\title{
The Effect of Lower Extremity Asymmetries on Low Back and Lower Extremity Pain with Pregnancy
}

\author{
Erica Casto
}

Follow this and additional works at: https://researchrepository.wvu.edu/etd

\section{Recommended Citation}

Casto, Erica, "The Effect of Lower Extremity Asymmetries on Low Back and Lower Extremity Pain with Pregnancy" (2016). Graduate Theses, Dissertations, and Problem Reports. 5323.

https://researchrepository.wvu.edu/etd/5323

This Thesis is protected by copyright and/or related rights. It has been brought to you by the The Research Repository @ WVU with permission from the rights-holder(s). You are free to use this Thesis in any way that is permitted by the copyright and related rights legislation that applies to your use. For other uses you must obtain permission from the rights-holder(s) directly, unless additional rights are indicated by a Creative Commons license in the record and/ or on the work itself. This Thesis has been accepted for inclusion in WVU Graduate Theses, Dissertations, and Problem Reports collection by an authorized administrator of The Research Repository @ WVU. For more information, please contact researchrepository@mail.wvu.edu. 


\title{
The Effect of Lower Extremity Asymmetries on Low Back and Lower Extremity Pain with Pregnancy
}

\author{
Erica Casto
}

Thesis submitted to the college of Health Sciences at West Virginia University in partial fulfillment of the requirements for the degree of

Master of Science in Exercise Physiology

\author{
Jean L. McCrory, PhD, Chair \\ Corrie A. Mancinelli, PT, PhD \\ Petronela Meszaros, MD \\ Division of Exercise Physiology \\ Department of Human Performance and Applied Exercise Science \\ Morgantown, West Virginia \\ 2016
}

Keywords: Pregnancy, pain, alignment, asymmetry, foot Copyright 2016 Erica Casto 


\section{ABSTRACT \\ The Effect of Lower Extremity Asymmetries on Low Back and Lower Extremity Pain with Pregnancy}

\section{Erica Casto}

Approximately $50 \%$ of pregnant women experience low back and lower extremity pain during pregnancy, many of which continue to experience pain postpartum. It is known that many women experience changes in foot size, arch height and lower extremity alignment. However, the mechanism by which these changes are related to pain is relatively unknown, specifically in regard to asymmetric changes in alignment. Therefore, the purpose of this study was to assess alignment during pregnancy in order to determine if asymmetries occurring in women during pregnancy are related to low back, hip, knee, and foot pain. Methods: Ten women in their third trimester of pregnancy and nine nulliparous controls were recruited to participate. Biomechanical measures of foot length, foot width, arch index, arch height index, arch rigidity index, arch drop, and rearfoot angle were taken bilaterally to assess asymmetry. Pelvic asymmetries were assessed in the frontal and sagittal plane. Musculoskeletal pain was assessed using a Visual Analogue Scale. Pregnant women were placed into a 'pregnant pain' group ( $n=5)$ and a 'pregnant no pain' group $(n=5)$ based on results of this scale. Results/Conclusion: A relationship between lower extremity asymmetries and pain during pregnancy does exist. Specifically, negative correlations were found between arch index asymmetry and low back pain $(p=0.005)$, foot length asymmetry and lower leg pain $(p=0.008)$, and pelvic obliquity and lower leg pain $(p=0.020)$. Significant positive correlations were found between foot width asymmetry and knee pain $(p=0.028)$, as well as arch drop asymmetry and upper leg $(p=0.024)$, knee $(p=0.005)$, and lower leg pain ( $p=0.019)$. This study was successful in identifying a few target areas for clinicians to treat pain, but requires a much larger sample size in order to establish differences between pregnant women who experience no pain and pregnant women who do experience pain. Because low-back and lower extremity pain is extremely prevalent in post-partum women, it is important to conduct further research in order to determine both whether these asymmetries are related to pain post-partum, and if treating these asymmetries is preventative of pain post-partum. 


\section{Contents}

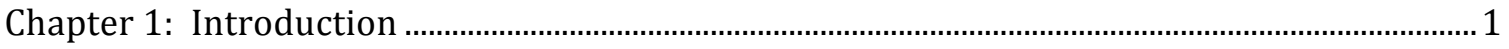

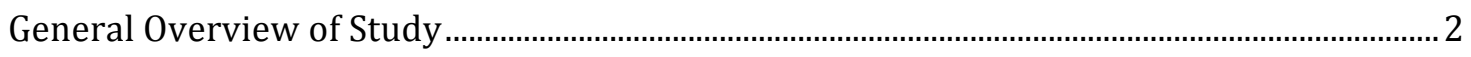

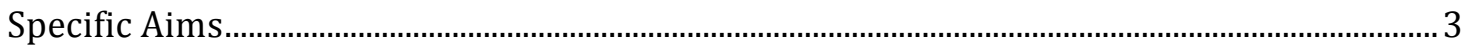

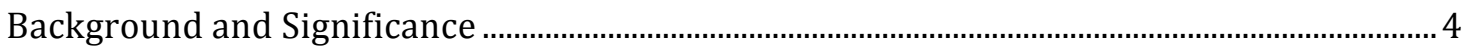

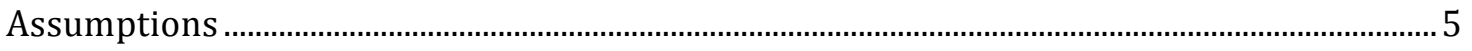

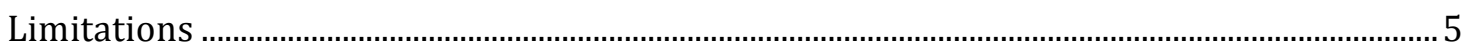

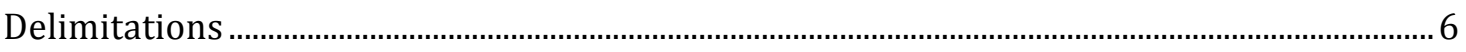

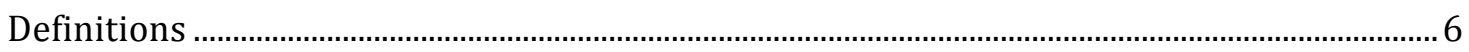

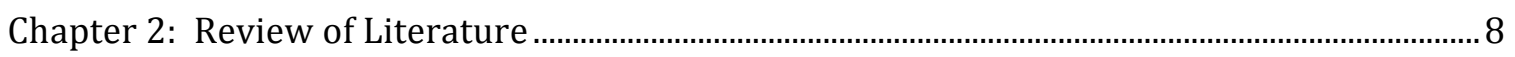

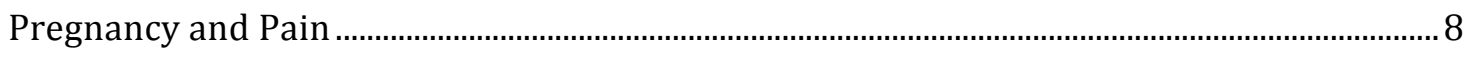

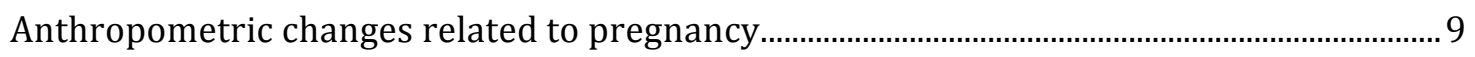

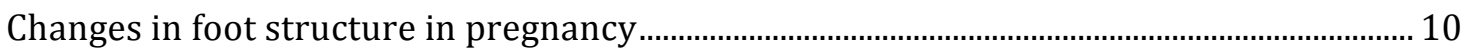

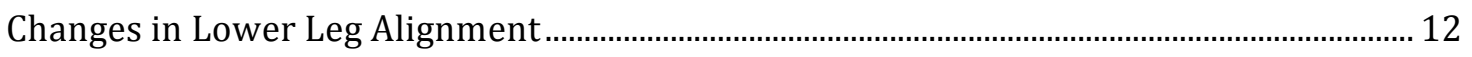

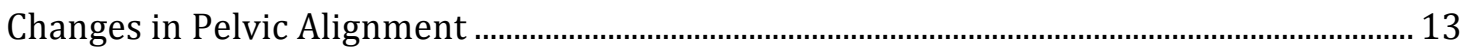

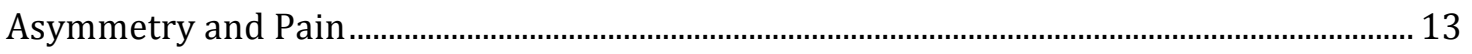

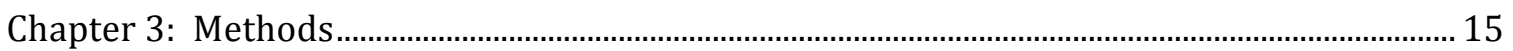

General Outline

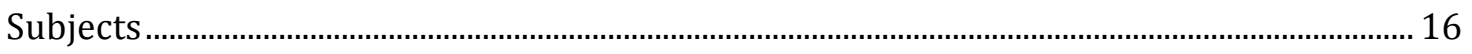

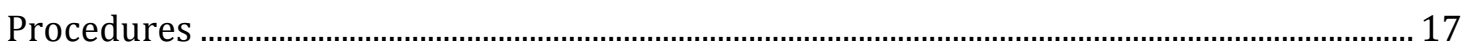

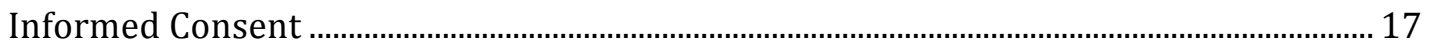

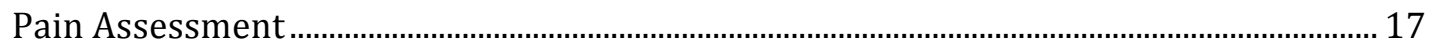

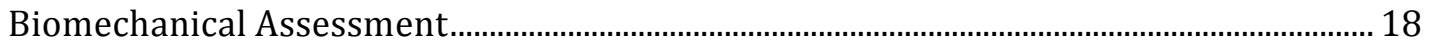

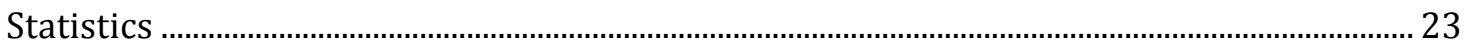

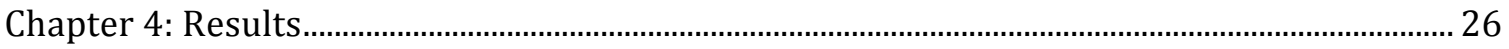




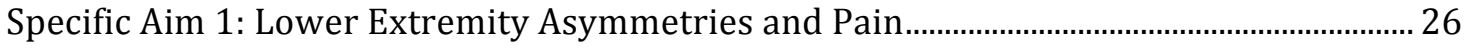

Specific Aim 2: Location of Pain and Alignment Asymmetries ................................................. 29

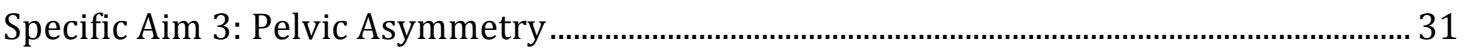

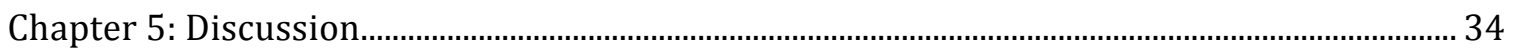

Specific Aim 1: Lower Extremity asymmetries and Pain ....................................................... 35

Specific Aim 2: Location of Pain and Alignment Asymmetries .................................................. 37

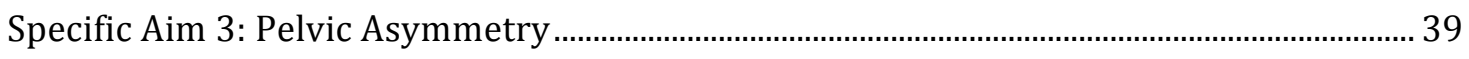

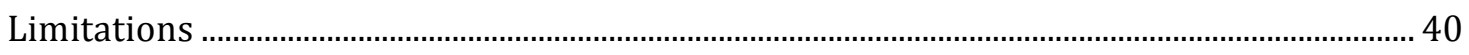

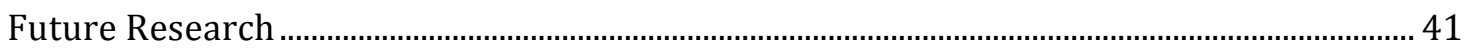

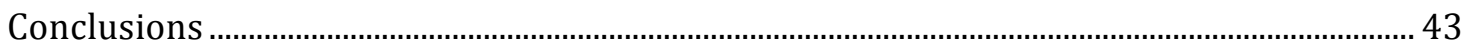

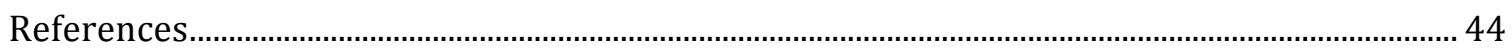

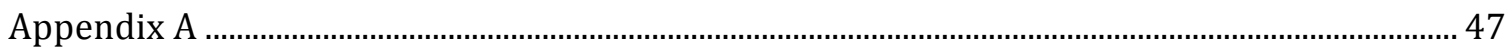

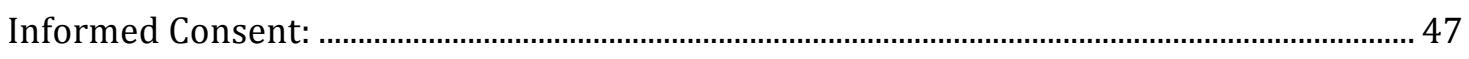

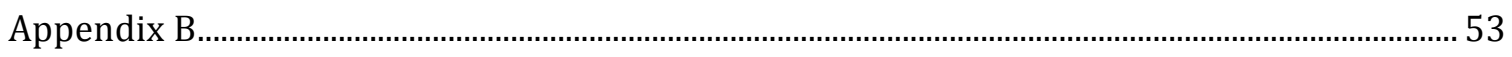

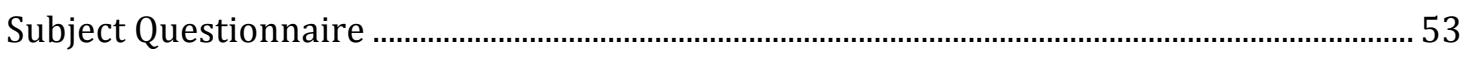

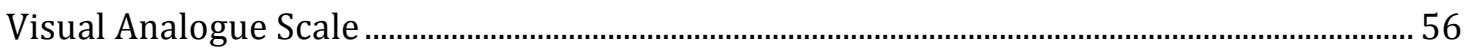

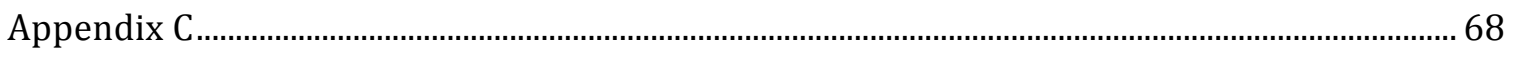

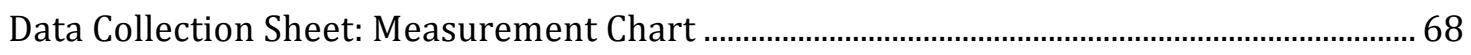

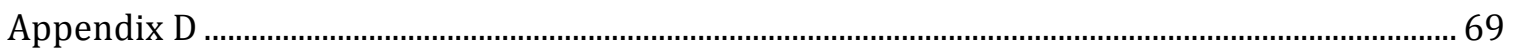

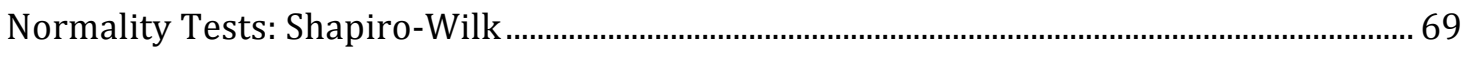




\section{Chapter 1: Introduction}

Lower extremity and low back pain is a common complaint among pregnant women (28). Increased pain can likely be attributed to the anatomic and physiological changes that occur throughout pregnancy (30). Among these changes are most notably increased abdominal volume, altered thoracopelvic alignment, and increased joint-laxity $(5,24-26)$. This increased joint-laxity is mediated by Relaxin, a hormone involved with endometrial maintenance and increasing ligamentous laxity to allow for pelvic girdle expansion $(5,17,31)$.

Relaxin, which spikes during early pregnancy, targets the ligaments of the pelvis in order to increase width of the symphysis pubis and sacroiliac joint (19). This creates a "cradle" for the fetus during pregnancy (19). However, Relaxin has been found to affect peripheral joint ligaments as well (5). Pregnancy-related ligamentous laxity has been correlated with changes in foot structure, including decreased arch height, increased foot width, and increased total foot length $(27,32)$. However, pregnancy alone was not the main predictor of foot shape alteration; significant increases in BMI combined with pregnancy were indicative of the greatest changes $(7,11)$.

Although previous studies have shown dimensional changes in the feet during pregnancy $(3,11,18,30,32)$, there is limited quantification of how these specific changes correlate to pain. Harrison (18) measured foot and lower extremity alignment alterations over the course of a first pregnancy and found a moderate correlation $(r \approx 0.3)$ with pain (18). However, because most of their study participants reported only low 
levels of pain, true correlations of lower extremity and foot alignment changes with pain could not be assessed (18).

Therefore, the purpose of this study was to assess alignment during pregnancy in order to determine if asymmetries occurring in women during pregnancy are related to low back, hip, knee, and foot pain. These asymmetries were compared to those of a non-pregnant control group. It is expected that these results will be useful to clinicians in the treatment of pregnant women experiencing pain, as well as the prevention of long-term pain.

\section{General Overview of Study}

Ten pregnant women in their third trimester of pregnancy, and nine nulliparous, non-pregnant women were recruited from the greater Morgantown area. After consent was obtained, subjects completed the pain assessment questionnaires. Pain was assessed using a Visual Analogue Scale (VAS) for the low back, posterior pelvis, and the right and left leg and foot. Pregnant subjects who reported pain $>3 / 10$ were placed into the pregnant pain (PP) group. Pregnant subjects with no pain (VAS $<3 / 10$ ) were placed in the pregnant no pain (PNP) group. Lastly, healthy non-pregnant women comprised a control group. We assessed the following parameters: foot length (FL), foot width (FW), Arch Index (AI), Arch Height Index (AHI), Arch Rigidity Index (ARI), Arch Drop (AD), Subtalar joint (rearfoot) angle (RFA), Pelvic Obliquity (PO), and Beighton's Test. 


\section{Specific Aims}

Specific Aim 1: To quantify foot and pelvic asymmetries in women with low back, posterior pelvic, leg and foot pain during pregnancy compared to a control group of pregnant women without pain, and a non-pregnant control group. Specifically, we examined the extent to which foot length, width, arch index, arch height index, arch rigidity index, arch drop, subtalar joint angle, and pelvic obliquity differ between limbs in pregnant women with pain, pregnant women without pain, and non-pregnant controls. Beighton's test of flexibility was also assessed between groups.

Hypothesis 1: We hypothesized that pregnant women with pain would have increased asymmetries in foot length, foot width, arch index, arch drop, subtalar joint angle, pelvic obliquity, and arch height index in comparison to pregnant women without pain, and the non-pregnant control group. We also hypothesized that pregnant women with pain would have increased Beighton's test scores.

Specific Aim 2: To examine the relationship of biomechanical measures of alignment to self-reported measures of foot, posterior pelvic, and lumbar spine pain in pregnant women with pain, pregnant women without pain, and a non-pregnant control group. Hypothesis 2: We hypothesized that biomechanical asymmetry in the pelvis and in the foot would be related to the occurrence and severity of foot, posterior pelvis, and lumbar spine pain.

Specific Aim 3: To examine the effect of pelvic girdle asymmetry on the incidence of pain in all subjects. 
Hypothesis 3: We hypothesized that subjects with asymmetries in the pelvic girdle will be more likely to be classified as having pain that those without pain.

\section{Background and Significance}

Pregnancy-related ligamentous laxity, caused by presence of the hormone Relaxin, has been reported to increase mobility of not only the pelvic joints, but peripheral joints as well $(5,22,30,31)$. This laxity is related to alterations in foot and lower extremity alignment in pregnant women $(18,32,37)$. However, due to the increases in foot volume often reported throughout pregnancy, some of these measured changes in foot posture remain suspect $(3,18,30,32)$. Limited data exist on the changes in lower extremity alignment throughout pregnancy in relation to pain (18), as well as the relationship between increased foot volume and pain.

Pelvic and low back pain is a very common complaint among pregnant women (29). Specifically, about half of all pregnant women report low back pain as well as pelvic girdle pain, while one-third report posterior pelvic pain during pregnancy $(4,28,29,35)$. Furthermore, more than half of women report lower extremity pain post-partum (35). This is likely related to changes in pelvic, lower extremity, and foot alignment as well as foot swelling during pregnancy.

Limited research has been conducted to quantify alterations and malalignments occurring in foot, leg, and sacroiliac alignment during pregnancy in relation to selfreported low-back, pelvic, and lower extremity pain, specifically with regard to asymmetry of alignment. More research in the area may lead to development of 
treatments to prevent such pain from occurring, either by orthotic and footwear interventions or physical therapy and exercise programs. Due to the high reported rate of women who experience long-term pain postpartum, this type of prevention is of significant concern.

\section{Assumptions}

In this study, assumptions were made in regard to the self-reported values in the pain questionnaires. It was assumed that participants answered accurately and honestly when describing and quantifying the pain they experienced. It was also assumed that the changes measured occur due to pregnancy and not outside influence. Lastly, it was assumed that these biomechanical measurements reflected change in alignment, as it was not possible to determine pre-existing malalignments.

\section{Limitations}

Limitations of this study include many lifestyle factors that could not be controlled outside of the laboratory setting such as activity level and overall health prior to and during pregnancy that could affect presence of musculoskeletal pain. Due to the fact that we did not assess the women before they became pregnant, we could not be sure that all asymmetries were pregnancy related. However, we assessed a nonpregnant control group in order to determine the typical amount of asymmetries in women who have not been pregnant. 


\section{Delimitations}

Pregnant women with and without pain were selected for this study. According to clinical experience, some women do not experience pain until the third trimester. In order to properly place pregnant women into a pain or no-pain group, they were recruited in the third trimester. Because we are assessing pain occurring with pregnancy, women, pregnant or nulliparous, were excluded if pain was due to previous or current injury.

\section{Definitions}

Arch drop (AD)- The sitting arch height index minus the standing arch height index. Arch index (Al)- A ratio between the area of the midfoot over the total foot contact area, excluding the toes.

Arch height index (AHI)- The ratio between the foot height and the truncated foot length, from the back of the heel to the head of the first metatarsal.

Arch rigidity index (ARI)- The ratio between the sitting arch height index and the standing arch height index.

Multigravid- Women who have been pregnant multiple times.

Nulliparous- Never having given birth to offspring.

Pelvic asymmetry (PA)-The ratio that defines the slope between the Anterior Superior Iliac Spines (ASIS) and Posterior Superior Iliac Spines (PSIS) in the frontal plane(12).

Pelvic obliquity (PO)- Deviation of the pelvis from the horizontal in the frontal plane. Pelvic Torsion- Unilateral rotation of the pelvis in the sagittal plane. 
Primigravid- Women who are pregnant for the first time.

Rearfoot angle (RFA)- The angle in the frontal plane between the leg and posterior calcaneus while standing. 


\section{Chapter 2: Review of Literature}

\section{Pregnancy and Pain}

Approximately $50 \%$ of pregnant women report low back pain during pregnancy

(28). Many of these women report severe pain which decreases overall quality of life and disrupts the ability to accomplish normal activities, such as work and sleep (29). Ostgaard et al. (29) reported that this pain may likely be due to the abrupt increase in bodyweight with simultaneous decreased stability of the pelvis, although, this likely is not the sole cause as many other lower extremity alignment changes also occur $(3,7$, $11,14,18,30,32,37)$. Many pregnant women also report pelvic girdle ( $50 \%)$, posterior pelvic $(\sim 33 \%)$, and lower extremity and foot pain $(\sim 56 \%)(4,29,35)$. Posterior pelvic pain is reported to be of higher intensity during pregnancy, while low back pain tends to persist post-partum (29).

The hormone Relaxin is responsible for increased laxity of the symphysis pubis and sacroiliac joint during pregnancy in order to prepare the mother for delivery of the fetus. This laxity is increased with an accumulative number of pregnancies (5). This may explain why pelvic pain is common during pregnancy. MacLennan et al. (23) reported a direct relationship between elevated levels of serum Relaxin and pelvic pain. Albert et al. (2) reported that risk of pelvic pain increases with number of pregnancies as well as the presence of low back pain. 
Several authors have reported on the relationship between postural alignment or joint laxity on low back pain. Franklin et al. (14) reported no relationship between specific postural alignment changes occuring during pregnancy, such as lumbar lordosis and anterior pelvic tilt, with low back pain. However, recent studies have shown a relationship between joint laxity and low back pain (22). Lindgren and Kristiansson (22) found that finger laxity early in pregnancy, specifically of the fourth finger, was associated with low back pain later in pregnancy and post-partum. Harrison (18) assessed hypermobility using Beighton's Ligamentous Laxity Scale and found that higher scores, indicative of more laxity in the joints, were found in non-pregnant control rather than pregnant subjects. This test takes into account six different areas of flexibility including extension of fingers beyond $90^{\circ}$, as well as flexion at the hip to touch to floor, hyperextension of the knees, touching the thumbs to the forearm, and hyperextension of the elbows. It is important to note that these could also be affected by swelling and increased abdominal size as the Beighton's scale takes into account more than just the laxity at the finger.

\section{Anthropometric changes related to pregnancy}

Many anthropometric changes occur throughout pregnancy including increased body mass, altered thoracopelvic alignment, and increased joint-laxity (5, 24-26). Increased body mass specifically in the abdomen has been associated with increased lumbar lordosis (14). The hormone Relaxin has long been associated with joint laxity in 
the pelvic region (5); however, Relaxin has been shown to increase laxity of peripheral joints as well, including in the hands and feet $(22,30,31)$.

\section{Changes in foot structure in pregnancy}

Pregnant women, often anecdotally, report an increase in foot size throughout pregnancy (37). However, multiple studies have verified that pregnant women do in fact experience increases in foot length and width $(3,18,32,37)$. While this is likely a result of increasing the ligamentous laxity within the foot, it is possible that these changes could be attributed to swelling $(3,37)$. In the general population, Dunn et al. (11) reported an increased incidence of arch collapse in women compared to men (9:1 ratio) (11). Given the higher rate of arch collapse in women, the authors speculated that this finding could be due to the effects of previous pregnancies on intrinsic foot ligaments (11).

Various authors have noted an increase in foot length during pregnancy $(18,32)$. Segal et al. (32) and Harrison (18) both reported significant increases in foot length in primigravid women with these changes remaining in effect post-partum. These results are consistent with the findings of Alvarez et al. (3), who reported increased foot length $(0.08 \mathrm{~mm})$, although their results were not statistically significant. This could be due to their measures on foot length being taken with a graph paper method rather than calipers or the $\mathrm{AHI}$ measurement system $(3,18)$.

Both Ponnapula and Boberg (30), and Wetz et al. (37) report increased foot width during pregnancy. Harrison (18) did not find significant changes in foot width. It is 
possible that sample size $(n=15)$ may be the cause for lack of significance in the study conducted by Harrison (18). Although Ponnapula and Boberg (30) reported significant increases in foot width, their study was qualitative and incorporated anecdotal survey data from pregnant women rather than caliper measurements.

Segal et al. (32) reported significant increases in arch drop and significant decreases in arch height and arch rigidity during pregnancy. Primigravid women experienced the most significant changes, suggesting that these changes are somewhat permanent (32). Harrison (18) did not find significant changes in arch drop or arch rigidity, although it was reported that the changes did tend to follow the same trend towards a more lax arch (18). This was stated to be due to lack of first trimester measurements (18).

Harrison (18) also reported an increase in arch index (AI) during pregnancy, which is a measure of the area of the midfoot, as a percentage of the total foot, that is in contact with the ground (6). In normal, non-pregnant, non-obese individuals, the Al is a relatively good representative of arch height (6). However, in pregnant women, because of the confounding factors of foot swelling and increased body fat, change in this measure may be more indicative of increased volume of the foot due to swelling or soft tissue gain $(3,30)$.

Both Wetz et al. (37) and Alvarez et al. (3) reported significant increases in foot volume during pregnancy. Wetz et al. (37) collected all volume measurements during pregnancy though, making it difficult to report possible cause of the increased volume as either edema or increased BMI. However, Alvarez et al. (3) compared volume 
measurements from the first and third trimester to post-partum measurements, and reported an $8.5 \%$ increase in volume on average, but only a $1.2 \%$ decrease by eight weeks postpartum compared to the third trimester. This suggests that the increased volume was only partially due to fluid retention and primarily due to soft tissue accumulation (3). This particularly raises suspicion to the reported increased in arch index as a measure of fallen arches during pregnancy.

It has been suggested that obesity lowers the longitudinal arch of the foot, and can result in flat foot deformity $(11,15)$. This must also be observed with caution as body composition, notably increased body weight, has been reported as a possible confounding factor in the interpretation of Al (36). Dunn et al. (11) report that almost half of all women experience a change in shoe size regardless of increases in BMI during pregnancy (11). This suggests that arch collapse may occur as a result of aging alone rather than increased weight or pregnancies (11). They did, however, find that while neither obesity nor pregnancy alone were significant predictors of increased shoe size, the two factors combined were significant predictors (11).

\section{Changes in Lower Leg Alignment}

Harrison (18) investigated other changes in alignment during pregnancy, including rearfoot angle. No significant changes were found in rearfoot angle throughout pregnancy, indicating no increases in foot pronation related to decreased arch height (18). It is important to note that these subjects were not in significant pain, so it may be possible that women experiencing significant pain may present significant 
changes in rearfoot angle. However, unrelated to pain, this may suggest that the increased arch index was due to increased volume rather than fallen arches. This is where the gap in the literature exists, as no current studies have examined the link between increased volume or increased pain with these measurements. It should also be noted that no current studies have compared these specific measures between limbs in order to determine malalignments in relation to pain in pregnant women.

\section{Changes in Pelvic Alignment}

Fann (13) assessed the postural asymmetry prevalence with relation to low back pain with regards to pelvic obliquity and lateral sacral angle in men and women. No significant results were found in these parameters; however, when pelvic obliquity was corrected in a clinic setting using heel lifts, most patients report a decrease in level of pain, suggesting there are other factors involved (13). Harrison (18) also investigated changes in frontal plane pelvic obliquity and found significant results. However, greater pelvic obliquity was found in controls and was associated with less pain in the foot and ankle, however no relationship for back pain has been established (18). The relationship between low back pain and pelvic obliquity cannot yet be established due to the small changes reported by the participants of that study (18).

\section{Asymmetry and Pain}

Harrison (18) reported data that support the relationship between asymmetry of alignment measures and musculoskeletal pain. It was reported that ARI asymmetry was correlated to pain in the upper leg and knee (18). A correlation between Al and pain was 
also noted (18), but no measures of asymmetries between limbs were noted. However, it must be distinguished that swelling may influence the Al measurements. The effects of asymmetry and pain during pregnancy have not been extensively studied.

Al-Eisa et al. (1) reported a relationship between low back pain and asymmetry in the pelvis of both men and non-pregnant women stating that those with low back pain had significantly higher pelvic asymmetry ratios. This has been debated in the literature as other studies have reported no positive association between low back pain and pelvic asymmetry (21). Herrington (20) concluded that ASIS height (upslip) must have an asymmetry of $>5 \mathrm{~mm}$ or 2.5 degrees before it can be considered clinically significant. As for pelvic torsion, it was reported that an average of $6-7^{\circ}$ was normal, with an average difference between sides of $<0.5^{\circ}(20)$. However, pregnant subjects were specifically excluded from these studies, while men were included. It is possible that women experience these asymmetries to a greater degree, especially with pregnancy.

Interestingly, Damen et al. (10) reported no association between sacroiliac joint laxity and pelvic pain during pregnancy; rather, they found that asymmetric laxity was associated with increased levels of pelvic pain. They later reported that moderate to severe pain was predictive of pain persistence post-partum (10). This warrants further research into the effects of asymmetric alignments rather than overall changes in alignment in relation to pain and persistence of pain post-partum. 


\section{Chapter 3: Methods}

\section{General Outline}

We recruited 10 pregnant women in their third trimester and 9 never-pregnant women from the greater Morgantown area. After consent was obtained (Appendix A), subjects completed the pain assessment questionnaires. Pain was assessed using a Visual Analogue Scale (VAS) at the low back, hip/buttocks, upper leg, knee, lower leg, and foot/ankle for each side. Pregnant subjects who reported pain $>3 / 10$ in any location were placed into the pregnant pain (PP) group. Pregnant subjects with no pain (VAS <3/10) at any locations were placed in the pregnant no pain (PNP) group. Lastly, healthy non-pregnant women comprised a control group. We then assessed the following parameters: foot length (FL), foot width (FW), Arch Index (AI), Arch Height Index (AHI), Arch Rigidity Index (ARI), Arch Drop (AD), Subtalar joint (rearfoot) angle (RFA), Pelvic Obliquity (PO), and Beighton's Ligamentous Laxity Scale.

Subjects reporting chronic pain or with known diagnosed scoliosis pre-pregnancy or in the control group were excluded from the study in order to avoid factors contributing to these asymmetries outside of pregnancy. Control subjects were nonpregnant women who have never had a previous pregnancy. The pregnant pain and non-pain groups included women with any number of pregnancies. 


\section{Subjects}

We recruited 19 women ages 21-34 in the Morgantown area. Ten pregnant women, primigravid or multigravid, were included and placed into a pain or no pain group. Nine participants were nulliparous and placed into a control group. Subjects were recruited using advertisements for the study in the Health Sciences Center and WVU Healthcare facilities. We recruited pregnant women from the clinical obstetrics practices at WVU Healthcare. All subjects were excluded with a reported history of lower extremity fractures or surgeries, ankle or knee sprains within a year, medical conditions affecting sensation, diabetes, and smoking. Each woman, once recruited, was asked to wear their own snug fitting clothing for data collection. Demographic data are shown in Tables 1 and 2.

Table 1: Demographics for Pregnant and Control Groups.

\begin{tabular}{|l|c|c|c|}
\hline & Control & Pregnant & p-value \\
\hline Age(yrs) & $22.0 \pm 1.1$ & $29.6 \pm 3.0$ & 0.07 \\
\hline Height(cm) & $162.7 \pm 4.6$ & $165.7 \pm 6.6$ & 0.590 \\
\hline Pre-Pregnancy Mass(kg)* & $62.4 \pm 6.4$ & $72.02 \pm 15.6$ & 0.001 \\
\hline
\end{tabular}

$*=p \leq 0.05$

Table 2: Demographics for Pregnant Pain and Pregnant No Pain Groups.

\begin{tabular}{|l|c|c|c|}
\hline & Pain & No Pain & p-value \\
\hline Age(yrs) & $29.4 \pm 4.2$ & $29.8 \pm 1.6$ & 0.147 \\
\hline Height(cm) & $167.1 \pm 8.1$ & $163.8 \pm 4.4$ & 0.160 \\
\hline Current Mass(kgs) & $88.1 \pm 13.8$ & $78.6 \pm 13.3$ & 0.831 \\
\hline Pre-pregnancy Mass(kgs) & $75.6 \pm 16.1$ & $69.45 \pm 16.0$ & 0.818 \\
\hline Weeks Pregnant & $31.0 \pm 1.6$ & $32.0 \pm 2.0$ & 0.855 \\
\hline \# of Pregnacies & $1.8 \pm 1.8$ & $1.4 \pm 0.6$ & 0.136 \\
\hline
\end{tabular}




\section{Procedures}

\section{Informed Consent}

Data collection took place at WVU Healthcare at Cheat Lake, as well as the Human Performance Lab on the eighth floor of WVU's Health Sciences Center. All equipment was portable, so testing was conducted at the most convenient location for each subject. At the initial visit, the experimental protocol was explained to the subject and written informed consent (Appendix A), approved by WVU's Institutional Review Board for the Protection of Human Subjects was obtained. Subjects were asked to wear their own snug fitting clothing.

\section{Pain Assessment}

Subjects were surveyed about current pain in the left and right lower extremities through the use of a visual analogue scale (VAS) questionnaire. This questionnaire was designed based on the previously validated VAS Foot and Ankle(Appendix B) (33). A series of six questions were asked about pain and disability at each the lower back (LB), hip/buttocks (HB), upper left leg (ULL), upper right leg (URL), left knee (LK), right knee (RK), lower left leg (LLL), lower right leg (LRL) , left foot/ankle (LFA) and right foot/ankle (RFA). For each question, a $10 \mathrm{~cm}$ horizontal line was provided, on which the subject was asked to place a mark to indicate the severity of her symptoms, with the left indicating worst possible pain, and right indicating no pain. The distance from the leftmost point of the line to the mark was measured to the nearest millimeter, to give a score out of 10. The score for each of the six questions at each location was then added together and 
divided by 6 , to give a total score out of 10 at each location. Zero on this scale indicated no pain, and 10 indicated worst imaginable pain. Subjects with a score of 3/10or higher in any location were classified into a 'pain' group. Subjects with scores less than $3 / 10$ were classified in the 'no pain' group (18).

\section{Biomechanical Assessment}

First, the subjects were asked remove their shoes and weight was measured using a standard bathroom scale (2020W Mechanical Rotating Dial Scale, Taylor Precision Products, Oak Brook, IL). To assess pelvic, lower extremity, and foot alignment, a series of biomechanical measurements were taken. These measurements were taken bilaterally on the subjects. The left or right side were randomly selected to be assessed first. This data recording sheet can be found in Appendix C.

The subject stood in a standing position with feet shoulder width apart while foot length (FL) and foot width (FW) were measured using a standard anthropometer (Lafayette Instrument Company, Model 01291). FL was measured as the distance from the most anterior aspect of the foot to the most posterior aspect (Intrarater reliability Pearson correlation coefficient:>0.99). FW was measured as the distance between the most medial to the most lateral aspects of the forefoot (Intrarater reliability Pearson correlation coefficient:>0.94). Measurements were recorded in centimeters. Measurements were then taken of the contralateral foot. Leg length was measured with the subject standing with feet shoulder width apart. The distance in centimeters 
from the greater trochanter to the apex of the lateral malleolus of each leg was be recorded.

Next, to get a measure of hypermobility, subjects were assessed on a modified version of the Beighton Ligamentous Laxity scale. This scale involves a series of five tests for flexibility: hyperextension of the knees, touching the thumbs to the forearm, extension of the small finger beyond $90^{\circ}$ and hyperextension of the elbows. We excluded the sixth test from the Beighton Ligamentous Laxity scale, as flexion at the hip to touch the floor proves difficult in pregnant women due to their increased abdominal volume (18). Each task that the subject was capable of completing was given one point, for a score out of five possible points. The subject was not forced to stretch beyond their comfort level. The higher the score received, the higher the degree of laxity.

Arch index (Al), an indirect assessment of arch height, was measured according to the methods described by Cavanagh and Rodgers (6) (Intrarater reliability Pearson correlation coefficient:>0.896). Each subject was instructed to stand on a typical analog bathroom scale. She was then asked to place one foot an inkpad (Aetrex Harris Mat) located on the side of the scale so that her feet were $15 \mathrm{~cm}$ apart. The inkpad is designed such that the subject does not come in contact with the ink and no ink gets on the subject's

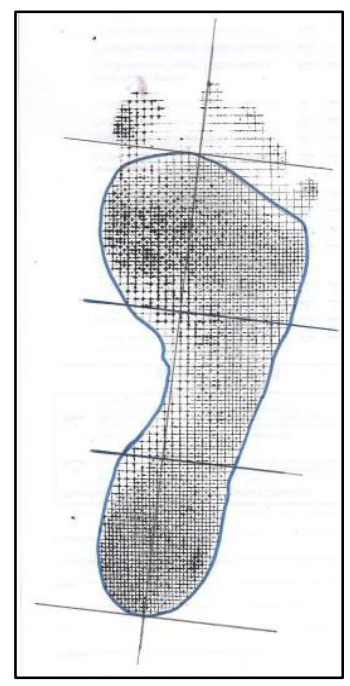

Figure 1: Arch Index 
foot. The subject was instructed to transfer her weight so that half of her weight was still be on the scale, and half of it was on the inkpad. An inked footprint was obtained in this manner. Al was calculated using the NIH software ImageJ (NIH, Bethesda, MD).

Specifically, on the footprint, a line was drawn from the tip of the second toe to the most posterior aspect of the heel. Distance from the anterior portion of the forefoot (not including the toes) to the posterior heel was measured along this line. This distance was divided into thirds and corresponding markers were placed along the line on the footprint. The most anterior portion of the footprint was termed the forefoot, and the middle and posterior regions were termed the midfoot and rearfoot, respectively. The areas of the midfoot and total footprint were then determined. Al was calculated as the area of midfoot / total area of the footprint(6). Increased Al indicates a lower arch, such that an $\mathrm{Al}<0.21$ is considered a high arch, an $\mathrm{Al}>0.26$ is considered a low arch, and an $\mathrm{Al}$ between 0.21 and 0.26 is considered normal (6). Measurements are made on both the left and right feet.

Several foot alignment measures were obtained using the Arch Height Index Measurement System (Intrarater reliability Pearson correlation coefficient: $>0.83)$. Using a set of sliding calipers (Figure 2. JAK Tool, New Jersey, New York), elevated on two wooden blocks to leave the medial longitudinal arch unsupported, three measurements of each foot were taken: foot length (FL), truncated foot length (TFL), which is the

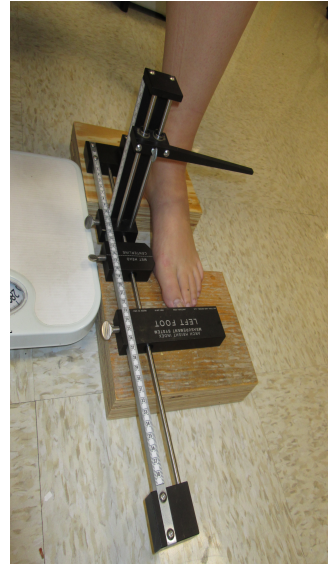

Figure 2: Arch

Height Index Measurement System distance from the most posterior aspect of the heel to the head of the first metatarsal, 
and foot height $(\mathrm{FH})$, which is the height of the foot at $1 / 2$ total foot length.

Measurements were taken seated, using a goniometer to place the ankle in a position such that the line between the first metatarsal head and the lateral ankle formed a $120^{\circ}$ angle with the line from the lateral ankle to the head of the fibula. Then the same measures were taken standing, with weight evenly distributed on both feet. Several calculations were made using these measurements. Seated arch height index $(A H I)=$ seated FH/seated TFL. Standing AHI = standing FH/standing TFL. Higher values of AHI indicate higher arches. Arch rigidity index (ARI) and arch drop (AD) are measures of arch flexibility. ARI = standing AHI/seated AHI. An ARI of 1 indicates a perfectly rigid arch, while values closer to 0 indicate a more flexible arch. $A D=$ seated $\mathrm{FH}$ - standing $\mathrm{FH}$. $\mathrm{A}$ greater AD indicates a more flexible arch.

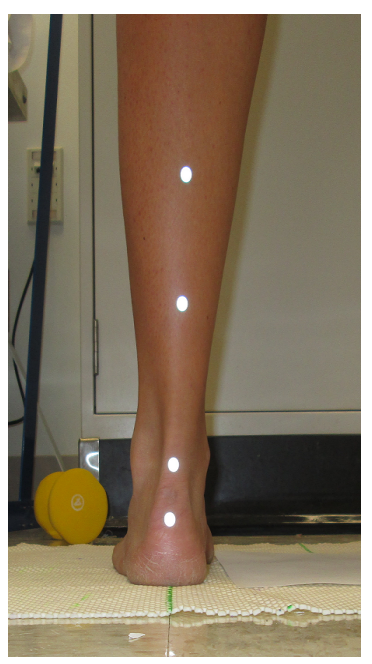

Figure 3: Rearfoot Angle
Rearfoot angle (RA) was also measured by photogrammetry, according to the methods of Clarke (8)(Fig.3). An anthropometer (Lafayette Instrument Company, Model 01291) was placed on the head of the fibula and the medial point on the leg directly across from the fibular head, and a line bisecting the knee joint line was dropped to locate and mark the midpoint of the subject's legs at the musculotendinous intersection of the gastrocnemius and on the Achilles tendon. While participants kneel on a chair facing the back, the subtalar joint was placed in a neutral position and marks were made on the midpoint of the calcaneus and Achilles. The subject was asked to stand in a relaxed position with feet 15 
$\mathrm{cm}$ apart. Reflective markers were placed on marked locations on both legs. A digital camera (Canon EOS Rebel T2i, Tokyo, Japan) was placed on a wooden block at a height of $10 \mathrm{~cm}, 55 \mathrm{~cm}$ directly behind the subject. A photograph was taken of both legs. Using ImageJ software, the rearfoot angle was calculated as the angle between the lines from the midpoint of the knee joint and the gastrocnemius insertion, and the gastrocnemius insertion and the midpoint of the calcaneus. Increased rearfoot angle indicates increased pronation. Measurements were obtained for both legs.

Pelvic obliquity (PO), or the angle that

the pelvis makes with the horizontal in the

frontal plane, was measured (Intrarater

reliability Pearson correlation coefficient: 0.96).

To do this, an experienced investigator palpated

the subjects' left and right anterior superior iliac

crests of the pelvis. Reflective markers $(1 \mathrm{~cm})$

were placed on these landmarks. The heights of

these markers were measured with an
A)

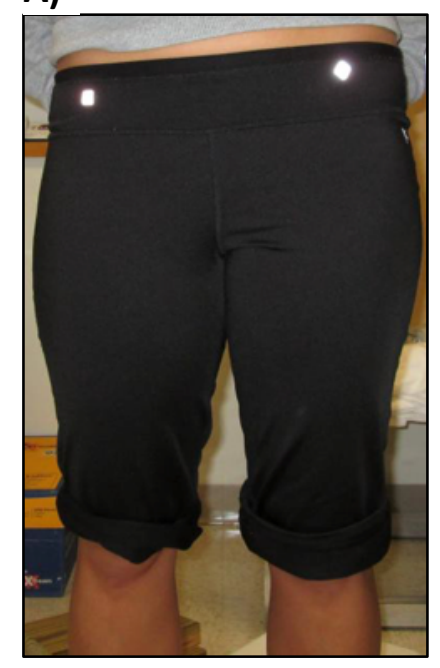

B)

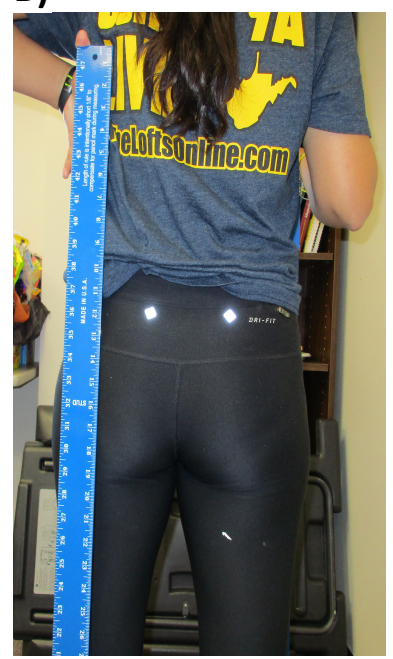

Figure 4: Pelvic Obliquity. A) PO using ASIS. B) PO using PSIS.

aluminum square ruler from the floor. The camera was positioned perpendicular to the subject. The subject was positioned with her feet $15 \mathrm{~cm}$ apart. A digital photograph was obtained (Figure 4). Using ImageJ software, pelvic obliquity, or the angle between a line connecting the left and right anterior superior iliac spine (ASIS) markers and the horizontal was determined. 
To assess pelvic asymmetry in the frontal plane (torsion), an additional photograph was taken with reflective markers on the posterior superior iliac spine (PSIS). Image-J was used to measure the angle of obliquity of the PSIS. The bilateral difference in degrees between both the PO of the ASIS and PO of the PSIS was then calculated.

To assess pelvic asymmetry in the sagittal plane (torsion), direct measurements of the height of the ASIS and PSIS were taken by measuring from the reflective markers to the apex of the medial malleolus. The distance between the ASIS and PSIS on the right and left sides were measured using an anthropometer (Lafayette Instrument Company, Model 01291). Calculations were performed with the given information to retrieve the angle of inclination on each side. The difference between these angles was a measure of pelvic torsion.

Finally, with the subject in a seated position, a test was performed to determine if the subjects had an aligned or unaligned pelvis. This was determined by placing the hand on top of each iliac crest and using the dominant eye to determine if the pelvis was even or uneven based on iliac crest (IC) height.

\section{Statistics}

Statistical analysis was performed using IBM SPSS Statistics software (Armonk, New York). Demographics (e.g. age, gender, height, weight) of the population were determined. Each subject was placed into a pregnant pain group (any pain >3), 
pregnant without pain group (pain $<3$ ), and non-pregnant control group. Descriptive statistics, including means and standard deviations, were calculated for continuous data. Specific Aim 1 was to quantify foot asymmetries in women with pain during pregnancy compared to a group of pregnant women without pain, and a control group of never pregnant women. Dependent variables included average bilateral differences for FL, FW, Al, AHI, ARI, AD, RA PO, and Beighton's test. Each measure of asymmetry was tested for normality using the Shapiro-Wilk test (Appendix D). The independent variable was the group (control, pregnant pain, pregnant no pain). An ANOVA was performed on each dependent variable to examine the difference between groups. Tukey post-hoc analysis was performed when appropriate $(\alpha=0.05)$.

Specific Aim 2 was to examine the relationship of self-reported measures of foot, posterior pelvic, and lumbar spine pain and biomechanical measures of alignment in all of the subjects. For this aim, we used correlation analysis to assess relationships between asymmetry and level of pain $(\alpha=0.05)$. Because these data were not normality distributed, Spearman-Rho non-parametric regressions were performed for each of the nine fluctuating asymmetry index calculations, as well as nine absolute difference calculations. These included ( $F L, F W, A I, A H I, A R I, A D, R A, P O$, and $\mathrm{PI}$ ), with pelvic pain, low back pain, leg pain, foot pain, and overall general pain for a total of 36 correlations for each FA and absolute difference measure.

Specific Aim 3 was to examine the effect of pelvic asymmetry on incidence of pain. Subjects were categorized as having pain or no pain. Subjects were classified as having an aligned or maligned pelvis due to IC height as well as pelvic torsion. To 
categorize IC height, subjects were said to have an aligned pelvis if their IC height was

even. They were said to have an unaligned pelvic is their IC height was uneven. Next, subjects were classified as having an aligned pelvis if they fell within 2 standard deviations of the mean pelvic torsion calculation of the control group (no torsion). They were said to have an unaligned pelvis if they fell outside of 2 standard deviations (torsion). A total of four chi-square analyses were performed for group and type of pelvic alignment $(\alpha=0.05)$. 


\section{Chapter 4: Results}

\section{Specific Aim 1: Lower Extremity Asymmetries and Pain}

Specific Aim 1 was to quantify foot asymmetries in women with pain during pregnancy compared to pregnant women without pain and a control group of never pregnant women. Dependent variables included the average differences for $\mathrm{FL}, \mathrm{FW}, \mathrm{Al}$, AHI, ARI, AD, RA, and PO. The score of Beighton's test is also included in Specific Aim 1. An ANOVA was performed on each dependent variable to examine the difference between groups.

No variables were significantly different between groups. However, mean asymmetry for $\mathrm{FW}, \mathrm{Al}, \mathrm{ARI}$, while not significant, presented interesting trends towards differences between the pregnant pain group, where the control and pregnant pain group appear to have no difference. FW, Al, and ARI asymmetries are presented in Figures 5, 6 and 7 respectively. The remaining variables are presented in Table 3. No significant relationship was found between groups for the modified Beighton's test for flexibility $(p=0.174)$. However, the mean Beighton's test score for the pregnant pain group was almost $86 \%$ lower than the pregnant no pain group (Figure 8). 


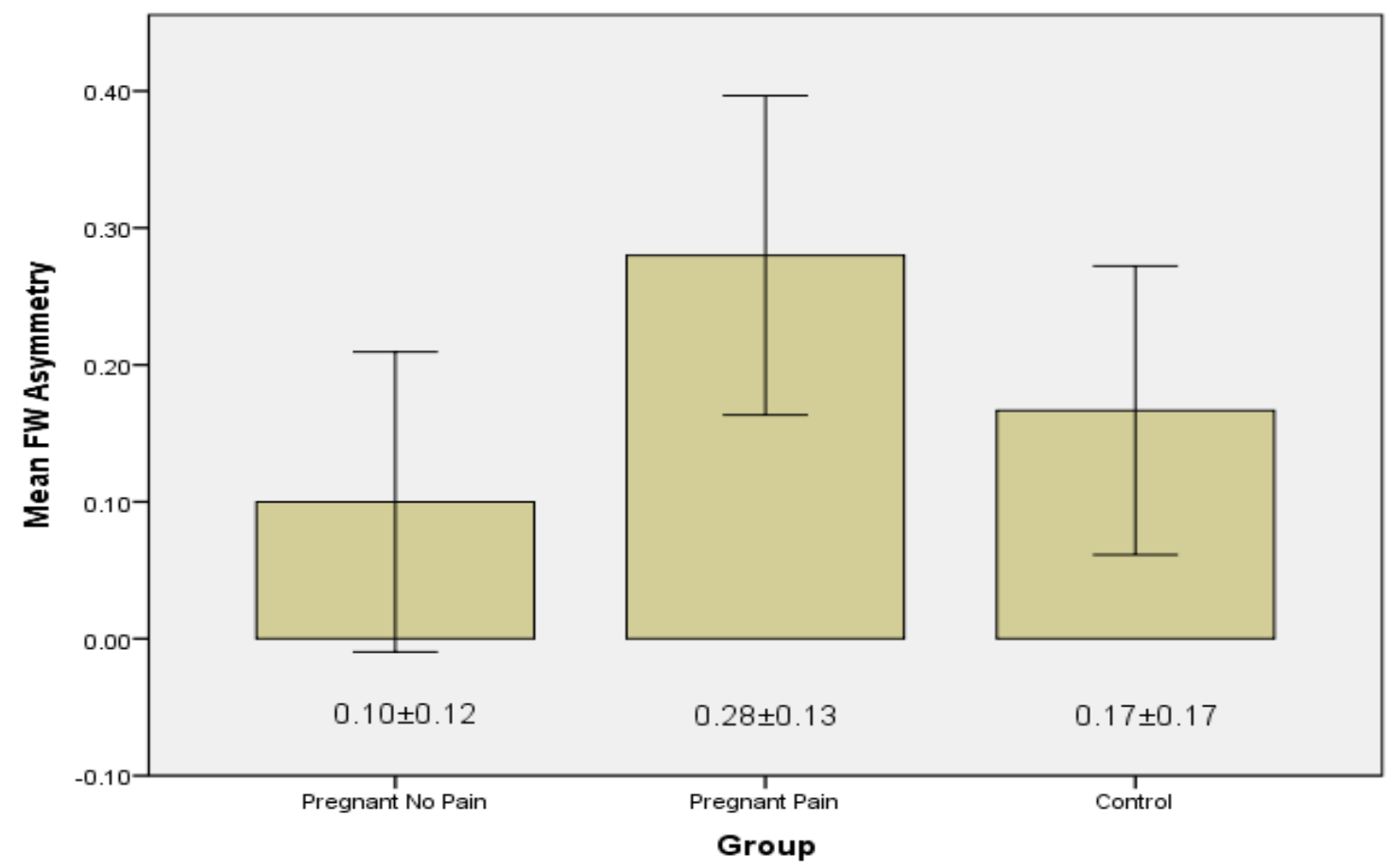

Figure 5: Mean Foot Width Asymmetry $(\mathrm{cm})$ for Pregnant No Pain, Pregnant Pain, and Control Groups. $p=0.163$.

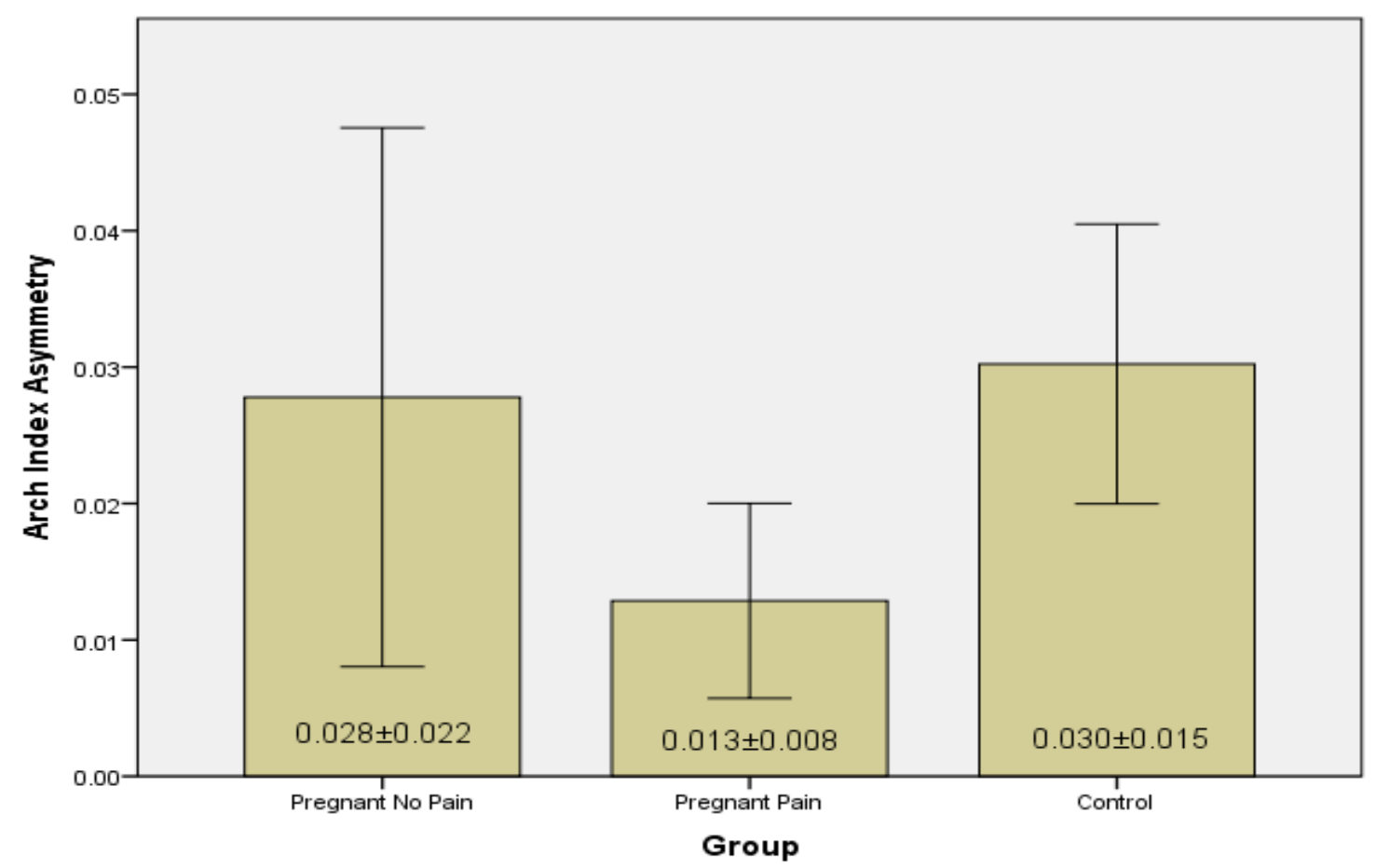

Figure 6: Mean Arch Index Asymmetries for Pregnant No pain, Pregnant Pain, and Control Groups. $p=0.169$. 


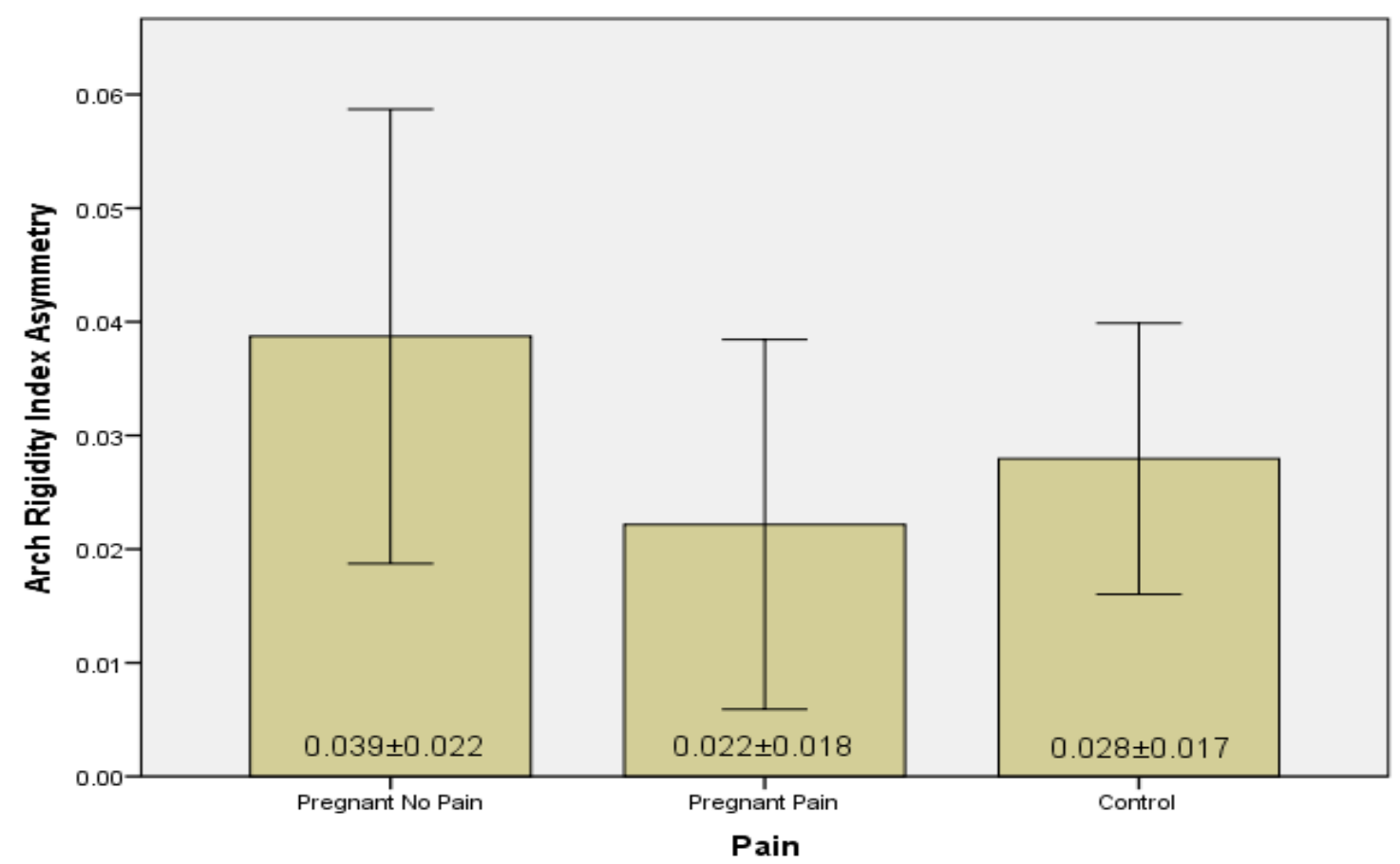

Figure 7:Mean Arch Rigidity Index Asymmetries for Pregnant No Pain, Pregnant Pain, and Control Groups. $\mathbf{p}=\mathbf{0 . 4 0 0}$.

Table 3: Lower Extremity Asymmetries with No Relationship to Pain Group

\begin{tabular}{|l|c|c|c|c|}
\hline Variable & $\begin{array}{l}\text { Pregnant No } \\
\text { Pain }\end{array}$ & $\begin{array}{l}\text { Pregnant } \\
\text { Pain }\end{array}$ & Control & p-value \\
\hline FL (cm) & $0.1 \pm 0.1$ & $0.22 \pm 0.30$ & $0.17 \pm 0.12$ & 0.588 \\
\hline AHI & $0.012 \pm 0.007$ & $0.008 \pm 0.007$ & $0.005 \pm 0.003$ & 0.078 \\
\hline AD (cm) & $0.14 \pm 0.26$ & $0.18 \pm 0.08$ & $0.11 \pm 0.21$ & 0.804 \\
\hline RA (degrees) & $2.23 \pm 1.23$ & $2.45 \pm 1.38$ & $2.26 \pm 1.51$ & 0.967 \\
\hline PO (degrees) & $2.54 \pm 1.52$ & $2.56 \pm 1.88$ & $2.14 \pm 1.20$ & 0.839 \\
\hline
\end{tabular}




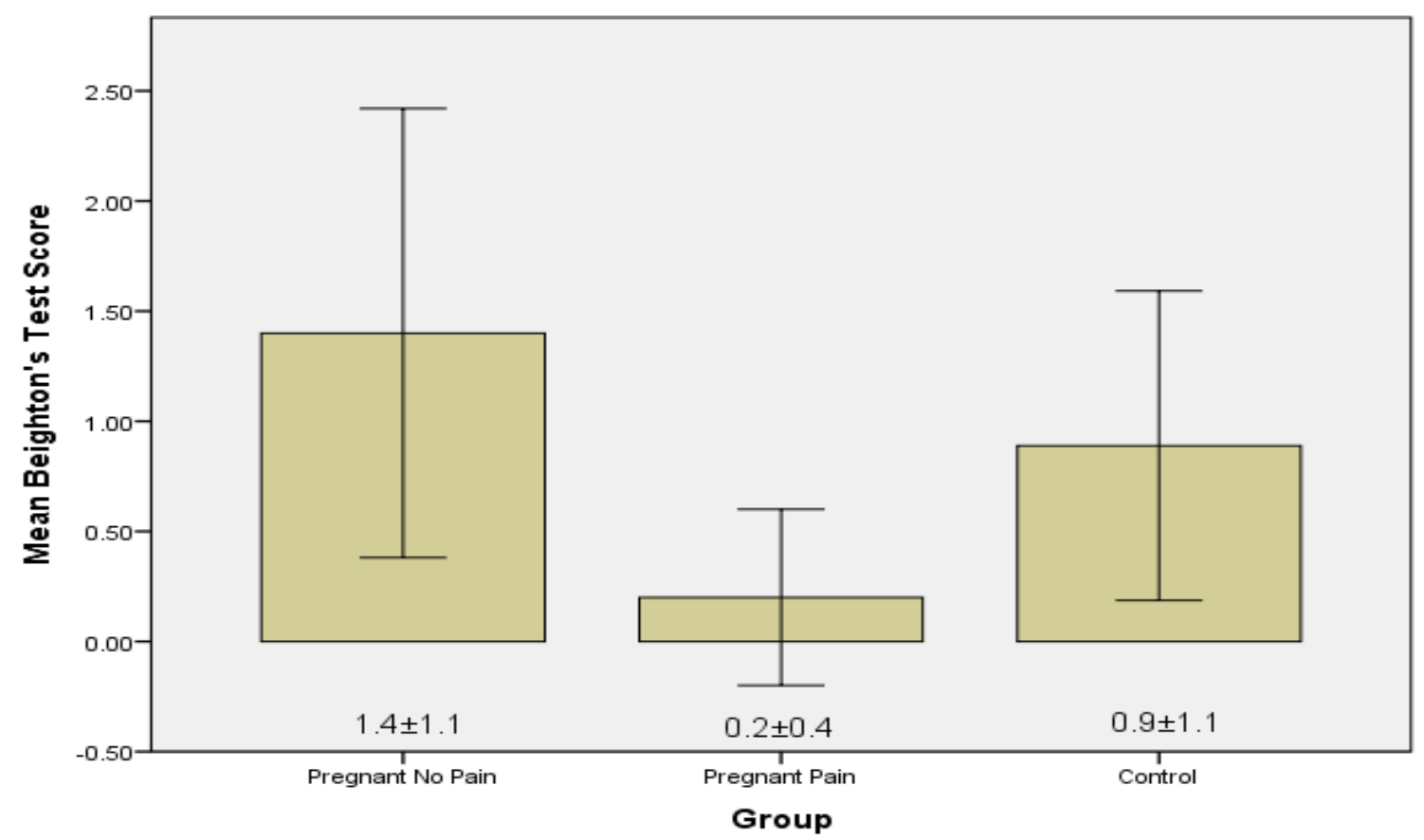

Figure 8: Mean Beighton's Test Scores for Pregnant No Pain, Pregnant Pain, and Control Groups. $p=0.174$.

\section{Specific Aim 2: Location of Pain and Alignment Asymmetries}

Specific Aim 2 was to examine the relationship of self-reported measures of foot, posterior pelvic, and lumbar spine pain and biomechanical measures of alignment in all of the subjects. Because the pain data were not normally distributed, a series of Spearman-Rho correlations, a non-parametric analysis, were performed to determine the correlation of each measure of asymmetry and each pain measure. Thus, SpearmanRho correlation coefficients were calculated for lower extremity asymmetries and low back, hip, upper leg, knee, lower leg, and foot and ankle pain (Tables 4 and 5).

Significant negative correlations were found between arch index asymmetry and low back pain (Table 4), foot length asymmetry and lower leg pain (Table 5), and pelvic 
obliquity and lower leg pain (Table 5). Significant positive correlations were found between foot width asymmetry and knee pain (Table 5), as well as arch drop asymmetry and upper leg (Table 4), knee (Table 5), and lower leg pain (Table 5).

Table 4: Spearman-Rho Correlation Coefficients for Low Back, Hip/Buttocks, and Upper Leg asymmetries and Pain

\begin{tabular}{|c|c|c|c|c|c|c|}
\hline \multirow{2}{*}{} & \multicolumn{2}{|c|}{ Low Back } & \multicolumn{2}{c|}{ Hip/Buttocks } & \multicolumn{2}{c|}{ Upper Leg } \\
\cline { 2 - 7 } & $\mathbf{R}$ & p-value & $\mathbf{R}$ & p-value & $\mathbf{R}$ & p-value \\
\hline FL & 0.127 & 0.61 & 0.085 & 0.729 & -0.271 & 0.262 \\
\hline FW & 0.370 & 0.119 & 0.402 & 0.088 & -0.104 & 0.672 \\
\hline Al & $-\mathbf{0 . 6 1 7}$ & $\mathbf{0 . 0 0 5 * *}$ & -0.390 & 0.099 & 0.017 & 0.945 \\
\hline AHI & -0.007 & 0.977 & 0.216 & 0.374 & -0.028 & 0.911 \\
\hline ARI & -0.141 & 0.566 & -0.190 & 0.436 & 0.247 & 0.308 \\
\hline AD & 0.280 & 0.246 & 0.097 & 0.693 & $\mathbf{0 . 5 1 4}$ & $\mathbf{0 . 0 2 4}$ \\
\hline RFA & -0.077 & 0.753 & 0.186 & 0.445 & 0.027 & 0.914 \\
\hline PO & -0.071 & 0.778 & -0.375 & 0.125 & -0.432 & 0.073 \\
\hline
\end{tabular}

$*=p \leq 0.05, * *=p<0.01$

Table 5: Spearman-Rho Correlation Coefficients for Knee, Lower Leg, and Foot/Ankle and Pain.

\begin{tabular}{|c|c|c|c|c|c|c|}
\hline & \multicolumn{2}{|c|}{ Knee } & \multicolumn{2}{|c|}{ Lower Leg } & \multicolumn{2}{|c|}{ Foot/Ankle } \\
\hline & $\mathbf{R}$ & $p$-value & $\mathbf{R}$ & $p$-value & $\mathbf{R}$ & p-value \\
\hline FL & -0.003 & 0.991 & -0.587 & $0.008^{* *}$ & -0.161 & 0.509 \\
\hline FW & 0.504 & $0.028^{*}$ & 0.302 & 0.208 & 0.396 & 0.093 \\
\hline Al & -0.321 & 0.181 & -0.006 & 0.982 & -0.171 & 0.484 \\
\hline AHI & -0.384 & 0.150 & -0.022 & 0.928 & 0.273 & 0.257 \\
\hline ARI & 0.291 & 0.226 & 0.313 & 0.191 & 0.182 & 0.457 \\
\hline AD & 0.619 & $0.005^{* *}$ & 0.534 & $0.019 *$ & 0.402 & 0.088 \\
\hline RFA & 0.323 & 0.177 & 0.243 & 0.316 & -0.081 & 0.742 \\
\hline PO & -0.105 & 0.677 & -0.542 & $0.020 *$ & -0.122 & 0.629 \\
\hline
\end{tabular}

$*=p \leq 0.05, * *=p<0.01$ 


\section{Specific Aim 3: Pelvic Asymmetry}

Specific Aim 3 was to examine the effect of pelvic asymmetry on incidence of pain. Chi Square analysis was used to compare the likelihood of a pregnant woman having an unaligned pelvis vs. a non-pregnant control using IC Height (Figure 9), as well using pelvic torsion (Figure 11). We also used a Chi Square analysis to test the likelihood of pregnant women in pain having an unaligned pelvis vs. a pregnant woman with no pain using IC Height (Figure 10), and pelvic torsion (Figure 12). No significant relationship was found between these pelvic asymmetries and pain.

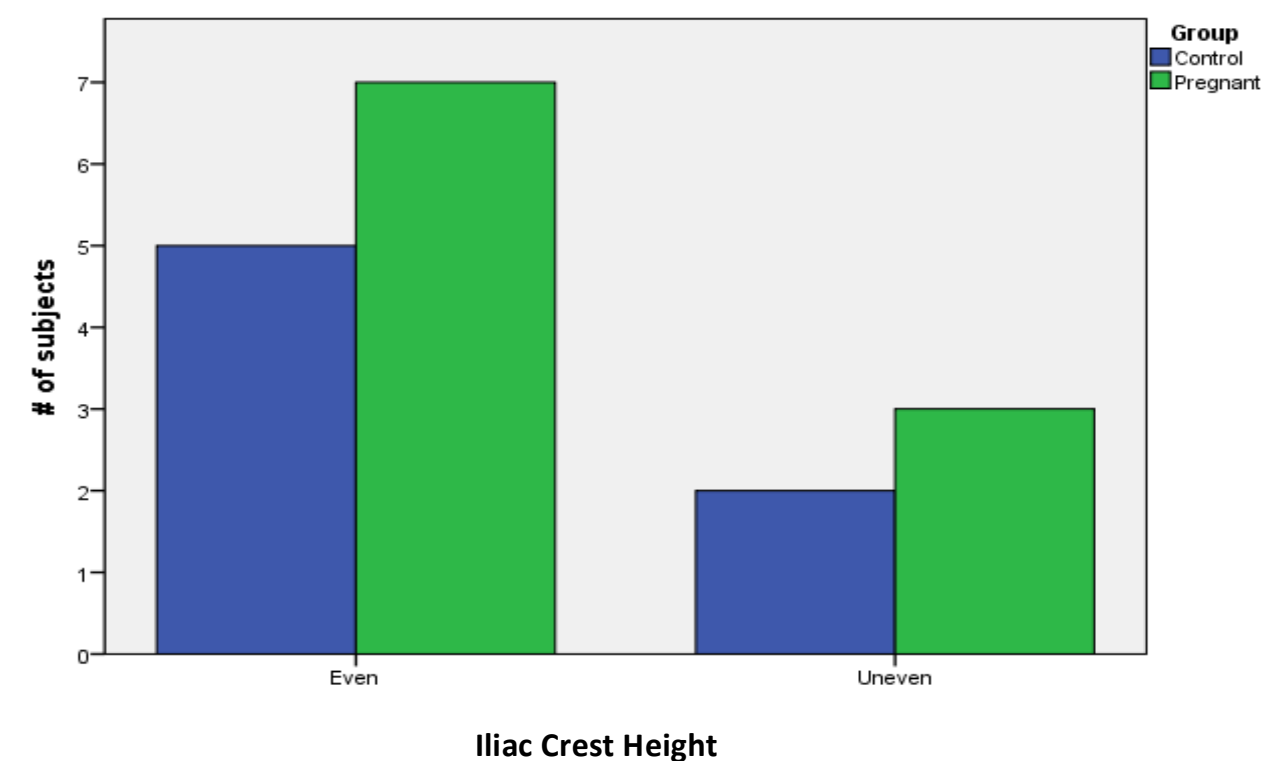

Figure 9: Chi Square analysis for Control $(n=7)$ and Pregnant Group $(n=10)$ with an aligned (even) vs. unaligned (uneven) pelvis using iliac crest height. $p=0.949$. 


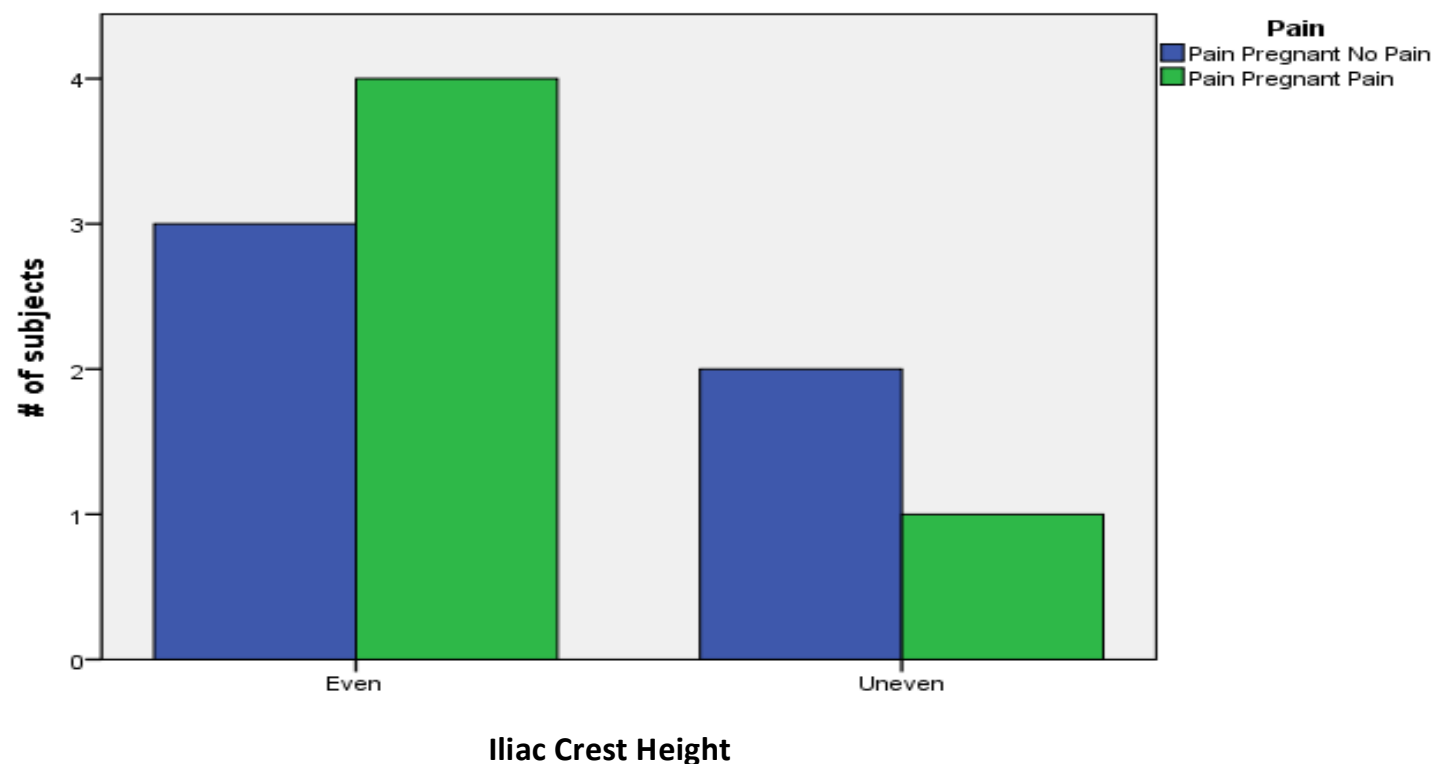

Figure 10: Chi Square analysis for Pregnant No Pain $(n=5)$ and Pregnant Pain $(n=5)$ groups with an aligned (even) vs. unaligned (uneven) pelvis using iliac crest height. $p=0.490$.

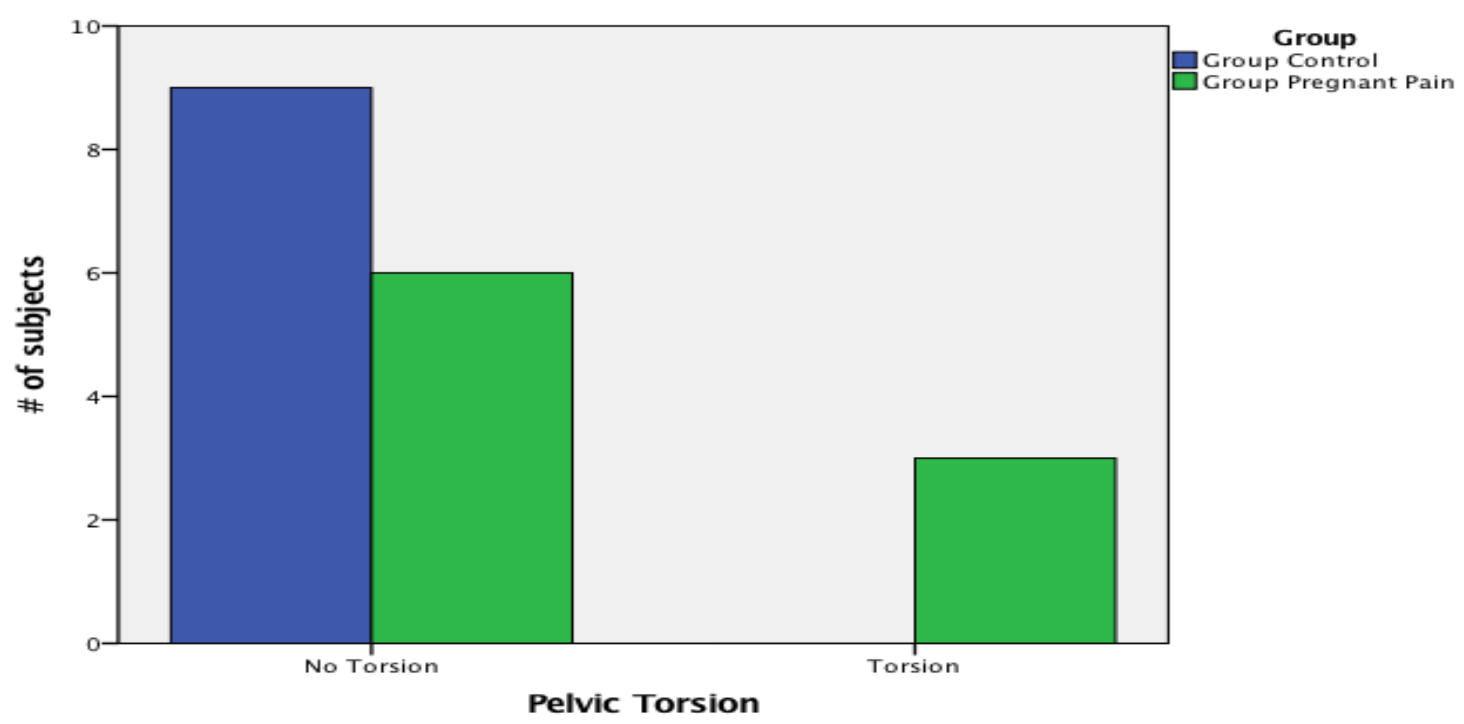

Figure 11: Chi Square analysis for Control $(n=9)$ and Pregnant Group $(n=9)$ with aligned (No Torsion) and unaligned (Torsion) pelvis using pelvic torsion calculation. $p=0.058$ 


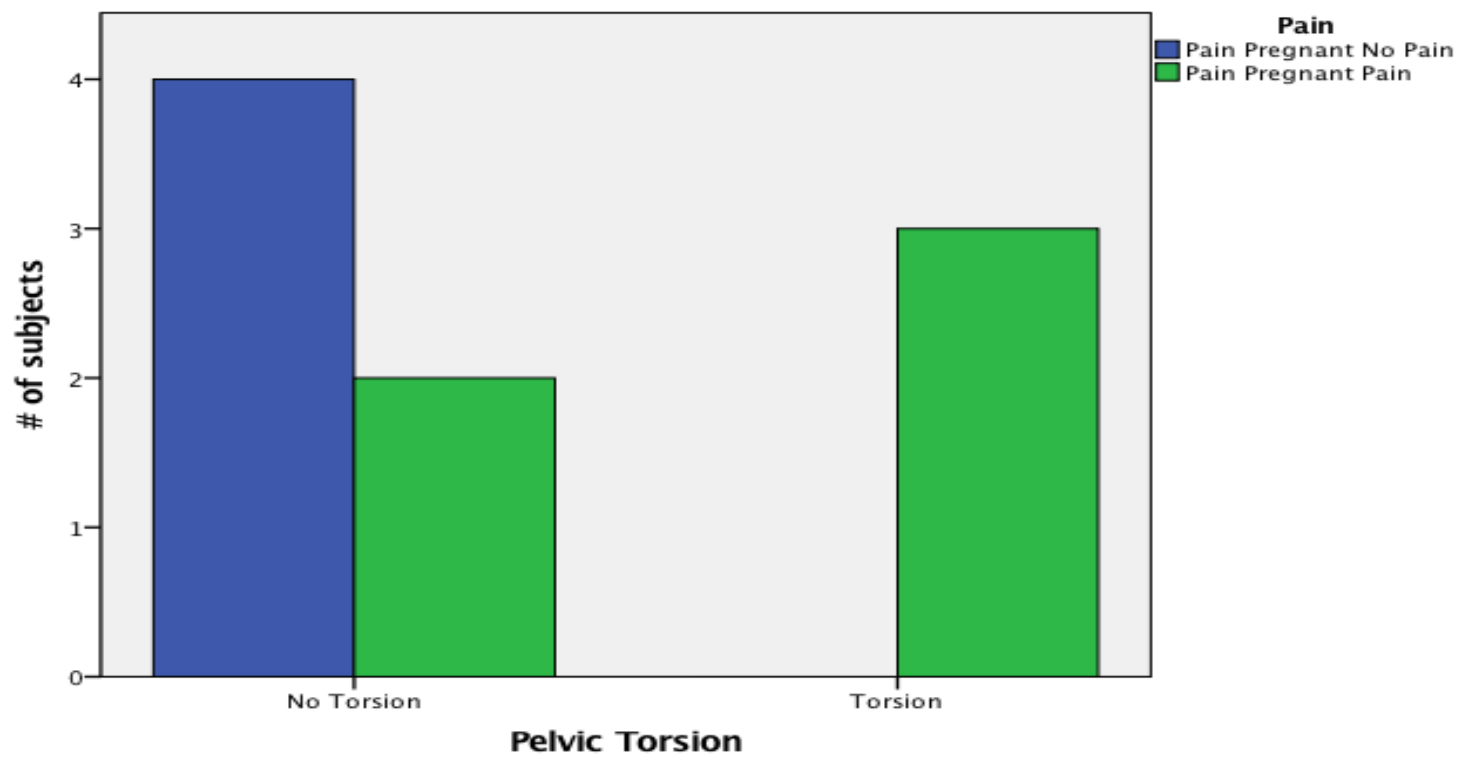

Figure 12: Chi Square analysis for Pregnant No Pain $(n=4)$ and Pregnant Pain $(n=5)$ groups with an aligned (No Torsion) vs. unaligned (Torsion) pelvis using pelvic torsion calculation. $\mathrm{p}=\mathbf{0 . 0 5 8}$. 


\section{Chapter 5: Discussion}

The purpose of this study was to assess alignment asymmetries during pregnancy in order to determine if these asymmetries were related to low back, hip, knee, and foot pain. Many pregnant women experience changes in alignment throughout the course of a pregnancy $(3,11,18,31)$, but these changes alone have not been strongly linked to pain (18). Therefore, we aimed to assess asymmetric alignment to determine its relationship to pain in three ways. First, we aimed to quantify the extent to which lower extremity asymmetries differ in pregnant women with low back, posterior pelvic, leg and foot pain, compared to pregnant women without pain and nonpregnant controls. Next, we examined the relationship of biomechanical measures of alignment asymmetries and the degree of self-reported measures of lower extremity and pelvic pain in pregnant women with pain, pregnant women without pain, and a non-pregnant control group. Finally, we assessed the effect of pelvic girdle asymmetries on the incidence of pain in all subjects.

In this study, $50 \%$ of pregnant women were experiencing low back pain, $40 \%$ were experiencing hip/buttocks pain, and $20 \%$ were experiencing knee, and foot and ankle pain. Reports of lower extremity pain in this study were slightly below the averages reported in previous literature of $\sim 56 \%(4,29,35)$. However, our sample is representative of the population of pregnant women experiencing low back pain as it is the same as what has previously been reported by Ostgaard (28) at $50 \%$. 


\section{Specific Aim 1: Lower Extremity asymmetries and Pain}

No lower extremity asymmetries were significantly different between pain groups, however, statistical power was very low. It is possible that we would see increased significance in the asymmetries for FW, Al, and ARI with an increased sample size. A power analysis for a $\beta \geq 0.8$ verifies that a reasonable sample size ( $n=52)$ would confirm whether a difference between groups exists for Al, while both FW and ARI would require even less. The data show a trend of the pregnant pain group having a larger FW asymmetry (Figure 5) when compared to both the pregnant no pain and control groups $(p=0.215)$. A sample size of at least 22 could give us enough power to observe this relationship. Previous examination of foot widening as a result of pregnancy are conflicting as Ponnapula and Boberg (30) and Wetz et al. (37) both reported increased FW, while Harrison (18) reported no changes. Harrison (18) had a very small sample size, and Ponnapula and Boberg (30) relied on anecdotal evidence. However, we assessed FW asymmetry rather than the change in FW over the course of a pregnancy. It should be noted that the trend towards pregnant women having a larger asymmetry could either be due to actual asymmetric changes, asymmetric swelling, or previous asymmetry in width such as the asymmetry that existed in the control group.

Likewise, the pregnant pain group shows a trend of a lower Al asymmetry than the other two groups $(p=0.301)$, but would likely require sample size of 52 participants to have enough statistical power (Figure 6). Al asymmetry should be taken with caution as these results may be heavily influenced by swelling. While the pregnant women had a lesser difference between sides, swelling could be the factor causing more contact with 
the ink pad in both feet. In the future this measure should be assessed with swelling taken into account by also taking measures post-partum.

ARI (Figure 7) shows a trend towards a higher ARI in the pregnant pain group that the other two groups, but would likely require sample size of at least 38 participants to have a large enough statistical power. Conversely, Segal et al. (32) reported a decreased ARI. However, these measures likely excluded swelling as a confounding factor because they were taken in the $1^{\text {st }}$ trimester and 19 weeks postpartum (32). Because arch rigidity is based on the flexibility of the foot, it is important to note that these measures could also be affected by swelling. Swelling could cause a "bottoming out" effect in the arch so the arches may appear rigid, when in fact they are just restricted in movement by the degree of swelling. This measure should be observed with swelling in the future in order to determine if the pain group is experiencing more swelling and if this factor does affect these measures.

No relationship could be established for $F L, A D$, and $R A$ asymmetries between these groups. Prior studies have reported increases in FL throughout pregnancy $(3,18$, 32); however, this asymmetric change had not been previously assessed. No asymmetric changes in $A D$ and RA are supported by no previous significant change in these measures throughout pregnancy (18).

Harrison (18) also reported no relationship between PO and pregnancy; however, a trend was noted that the control group had increased PO compared to the pregnant group. This trend could not be confirmed. Means for PO were similar across groups. We assessed iliac crest height with the subjects sitting down in order to 
determine if obliquity could be caused by a leg length discrepancy rather than actual pelvic malalignment. When using the categorical measure of iliac crest height, even or uneven, the number of subjects with uneven heights in each group was not significantly different from pregnant to control.

Our modified Beighton's Test scores yielded no significant results, however, the data trended $(p=0.153)$ towards the pregnant pain group having the least flexibility, while the pregnant no pain group were slightly more flexible that the control group. Harrison (18) reported results from the Beighton's test to be highest in the control group when compared to the pregnant women. However, this included bending over at the waist to touch the toes. This part of the test may have been significantly affected by the added abdominal mass, particularly to those women in their third trimester. By having taken out this measure, this may confirm the assumption made by Harrison (18) that the test results were heavily influenced by swelling which may have restricted their range of motion at joints.

\section{Specific Aim 2: Location of Pain and Alignment Asymmetries}

Spearman-Rho correlation coefficients were calculated to determine the relationship between asymmetry and pain, and seven significant correlations were found. Changes specifically occurring at the foot with the exception of AHI and ARI had a significant correlation to pain in specific areas. Our findings were consistent with Harrison (18) in that AD asymmetries were significantly correlated with upper leg, knee and lower leg pain, in that increased in this asymmetry were related to increased 
reported pain. Harrison (18) also noted significance in foot and ankle pain, but we could not confirm that finding. In fact we found no relationship between asymmetries and foot and ankle pain. Regardless, this is useful information in clinical treatment. It is possible that reducing the asymmetry in these specific areas can reduce the overall pain in targeted areas. However, a much larger sample size will be needed to confirm these findings.

Harrison (18) noted a correlation of ARI and upper leg pain, however, our study did not confirm this. In fact, ARI asymmetry was not related to pain in any area. When we assessed asymmetric Al we also found relation to pain. Specifically, there was a significant negative correlation $(p=0.011)$ between Al asymmetry and low back pain. Change in Al alone was related to pain in the study by Harrison (18), but asymmetry was not assessed. This makes sense considering that the pregnant pain group had the least amount of Al asymmetry. It is possible that less asymmetry exist in Al due to low arches having a "bottomed out" effect, such that the arches are as low as they can go. Again, however, we must take warning that swelling may have significantly affected this measure in both cases.

Nevertheless, these relationships established with areas of pain could be extremely useful in the clinical setting. Even with a small sample size, knee, lower leg, and low back pain had very significant correlations to AD asymmetries, FL asymmetries, and $\mathrm{Al}$ asymmetries respectively. It is important to note that every pregnant women in our pain group reported having low back pain as well as pain in at least one other area. 
Treating for these asymmetries could be useful to determine if the treatments can alleviate pain, and of particular importance, low back pain.

\section{Specific Aim 3: Pelvic Asymmetry}

No significant relationship was found between pelvic asymmetries and pain. This agrees with previous data reported by Levangie (21) stating that pelvic obliquity was not related to low back pain. Their study assessed obliquity using correlation (21). In our study, a Chi Square was used to assess the categorical data of iliac crest height. Iliac crest height was assessed while the subject was in a sitting position. By having the subject sit, we are able to rule out a leg length discrepancy to rule out the cause of obliquity.

Conversely, there was an almost significant trend towards pelvic asymmetry using the calculation of pelvic torsion and pain $(p=0.058)$. While the number of pregnant women in pain who had pelvic torsion and those who had no pelvic torsion were about even, it is very interesting that none of the pregnant women experiencing no pain had pelvic torsion. However, it should be noted that these measures of torsion may not necessarily exclude asymmetries occurring in the lower extremity causing pelvic obliquity rather than an actual malalignment of the pelvis in the way that our iliac crest height classification did. 


\section{Limitations}

Many limitations exist in this study. First, we had to use never- pregnant control subjects to represent the pre-pregnancy group. Our pregnant group was almost significantly older $(p=0.07)$ and significantly heavier pre-pregnancy $(p=0.001)$ compared to our never pregnant control group. Some of these measures could be individually varying as well as affected by age and weight rather than pregnancy.

Next, we were unable to attain post-partum measures due to time restrictions. This could have a huge effect on our results specifically in regards to swelling. In addition, with no post-partum measures, we are unable to know which, if any, women will continue to experience pain after giving birth.

The confounding effect of swelling is also a large limitation to this study. Many of our measures may have been affected by swelling. The degree of swelling from week to week often changes in the third trimester, and is also affected by the time of day. Some of our subjects came in first thing in the morning, but some were tested in the evening after they had worked 8 hours, which may have increased their degree of swelling. Also, our pain measures had to be self-reported as we cannot directly measure pain. For this reason, we may have varying levels of pain reported based on individual pain tolerance. It should also be noted that in both animals and humans there has been a reported increase in pain tolerance throughout pregnancy $(9,16)$. This may have severely impacted our pregnancy pain and no pain groupings as well as the correlations involving the degree of pain when comparing to never-pregnant controls. 
Pelvic measures were limited by the marker placement in the pregnant group. Markers could sometimes be difficult to see in the frontal plane. Photos were unable to be obtained in the sagittal plane, as the markers are not visible from this angle. Pelvic measures of torsion were classified categorically, rather than on a sliding scale. It should also be noted that pelvic obliquity may occur due to scoliosis. To our knowledge, no participants had scoliosis, but this is a possible limitation. Future studies should include a screening to test for this.

Finally, we were very limited by a small sample size as well as time to perform measurements. Because of our small sample size, we had very low power in statistical analysis. In addition, asymmetry measurements are subject to human error and therefore should be taken more than one time. However, due to time restrictions with the subjects and the number of measures being performed, we could only collect one measurement.

\section{Future Research}

Changes in alignment and the development of musculoskeletal asymmetries during pregnancy need to be explored further in order to determine targeted areas to prevent pain. Though we examined pregnant women who are in pain vs. those who were not, we had a very small sample size. It is possible that with a larger sample size, more significant relationships may be established.

Swelling should be assessed during pregnancy and post-partum. The degree of swelling experienced can be compared to the alignment measures as well as the degree of pain experienced. In this way it can be used as a covariate, rather than a limitation. If 
no post-partum measures will be acquired, foot volume should be measured for the control group as well with a similar BMI to the pregnant group pre-pregnancy BMI. This will help make the assumption that the pregnant group was in fact swollen.

Next, all measures of alignment should be taken at least three times. This way the average difference between sides can be used to determine fluctuating asymmetry. Fluctuating asymmetry shows less measurement error due to variation when an average of at least three measures is used (34). We were limited to only attaining one measurement due to time constraints. These women were standing for approximately one hour during testing, but reliability was good for each of these measures, so we were comfortable attaining only one measurement. For this reason, we chose to use the difference between sides rather than FA, however accuracy could be increased in the future with an average rather than one measure.

Finally, due to the increased pain threshold reported in pregnant women, it is possible that future studies should avoid the pregnant group all together in order to establish these relationships with pain. Rather, they could use a post-partum group in pain and not in pain to make these comparisons. Specifically, this post-partum group should be approximately 1-2 years post-partum in order to be sure these effects are permanent. This would not only eliminate some error in self-reports of pain, but also error which may have been caused by swelling. Eliminating the pregnancy group will allow an easier recruitment process when recruiting specifically for pain. This will likely significantly increase the sample size attained in the same time span, ultimately increasing the overall power in statistical analysis. Ultimately, this could provide a bigger 
picture of how to prevent pain occurring during pregnancy. This allows us to focus on the lasting pain, rather than any pain experienced throughout pregnancy.

If a relationship is established, this could be significant for future work regarding prevention of pain in women with pregnancy. This could help identify target areas for clinicians to treat pain using orthotics, as well as strengthening protocols to maintain structure and alignment of both the foot and lower extremity.

\section{Conclusions}

In conclusion, there is some relationship between lower extremity asymmetries and pain during pregnancy. This study was successful in identifying a few target areas for clinicians to treat pain, but requires a much larger sample size in order to establish differences between pregnant women who experience no pain and pregnant women who do experience pain. Because low-back and lower extremity pain is extremely prevalent in post-partum women, it is important to conduct further research in order to determine both whether these asymmetries are related to pain post-partum, and if treating these asymmetries is preventative of pain post-partum. 


\section{References}

1. Al-Eisa E, Egan D, Wassersug R. Fluctuating asymmetry and low back pain. Evolution and Human Behavior. 2004;25(1):31-7.

2. Albert HB, Godskesen M, Korsholm L, Westergaard JG. Risk factors in developing pregnancy-related pelvic girdle pain. Acta Obstetricia et Gynecologica Scandinavica. 2006;85(5):539-44.

3. Alvarez R, Stokes A, Asprinio D, Trevino S, Braun R. Dimensional changes of the feet in pregnancy. The Journal of Bone and Joint Surgery. 1988;70$\mathrm{A}(2): 271-74$.

4. Bergstrom C, Persson M, Mogren I. Pregnancy-related low back pain and pelvic girdle pain approximately 14 month after pregnancy- pain status, selfrated health and family situation. Pregnancy and Childbirth. 2014;14(48):112.

5. Calguneri M, Bird HA, Wright V. Chenges in Joint Laxity Occuring During Pregnancy. Annals of the Rheumatic Diseases. 1982;41(2):126-8.

6. Cavanagh PR, Rodgers MM. The Arch Index - A useful measure from foorprints. Journal of Biomechanics. 1987;20(5):547-51.

7. Chiou W-K, Chiu H-T, Chao A-S, Wang M-H, Yi LC. The influence of body mass on foot dimensions during pregnancy. Applied Ergonomics. 2015;46.

8. Clarke TE, Frederick EC, Hamill CL. The effects of shoe design parameters on rearfoot control in running. Medicine and Science in Sports and Exercise. 1983;15(5):376-81.

9. Cogan R, Spinnato JA. Pain and discomfort thresholds in late pregnancy. Pain. 1986;27(1):63-8.

10. Damen L, Buyruk HM, Güler-Uysal F, Lotgering FK, Snijders CJ, Stam HJ. Pelvic pain during pregnancy is associated with asymmetric laxity of the sacroiliac joints. Acta Obstetricia et Gynecologica Scandinavica. 2001;80(11):1019-24.

11. Dunn J, Dunn C, Habbu R, Bohay D, Anderson J. Effect of pregnancy and obesity on arch of foot. Orthopaedic surgery. 2012;4(2):101-4.

12. Egan DA, Cole J, Twomey L. An Alternative Method for the Measurement of Pelvic Skeletal Asymmetry (PSA) Using an Asymmetry Ratio (AR). Journal of Manual \& Manipulative Therapy. 1999;7(1):11-9.

13. Fann AV. The Prevalence of Postural Asymmetry in People With and Without Chronic Low Back Pain. Arch Phys Med Rehabil. 2002;83:1736-38.

14. Franklin ME, Conner-Kerr T. An Analysis of Posture and Back Pain in the First and Third Trimesters of Pregnancy. JOSPT. 1998;28(3):133-38.

15. Ganu S, Panhale V. Effect of obesity on arch index in young adults. Online Journal of Health and Allied Sciences. 2012;11(4):1-3.

16. Gintzler A. Endorphin-mediated increases in pain threshold during pregnancy. Science. 1980;210(4466):193-5. 
17. Goldsmith L, Gerson W. Relaxin in human pregnancy. In. Relaxin and Related Peptides: Fifth International Conference. New York, NY: Academy of Sciences; 2009, pp. 130-35.

18. Harrison K. Lower extremity alignment and back pain during pregnancy. Morgantown, WV: West Virginia University; 2014. 66 p.

19. Heckman JD, Sassard R. Musculoskeletal considerations in pregnancy. J Bone Joint Surg Am. 1994;76(11):1720-30.

20. Herrington L. Assessment of the degree of pelvic tilt within a normal asymptomatic population. Man Ther. 2011;16(6):646-8.

21. Levangie PK. The Association Between Static Pelvic Asymmetry and Low Back Pain. Spine. 1999;24(12):1234-42.

22. Lindgren A, Kristiansson P. Finger joint laxity, number of previous pregnancies and pregnancy induced back pain in a cohort study. Bmc Pregnancy and Childbirth. 2014;14.

23. MacLennan AH, Nicolson R, Green RC, Bath M. Serum relaxin and pelvic pain of pregnancy. The Lancet (British edition). 1986;2(8501):243-6.

24. McCrory JL, Chambers AJ, Daftary A, Redfern MS. Dynamic postural stability during advancing pregnancy. Journal of Biomechanics. 2010;43(12):2434-9.

25. McCrory JL, Chambers AJ, Daftary A, Redfern MS. Ground reaction forces during stair locomotion in pregnant fallers and non-fallers. Clinical Biomechanics. 2014;29(2):143-8.

26. McCrory JL, Seay JF, Hamill J. Thoracopelvic Coordination of Pregnant Women during Gait. Medicine and Science in Sports and Exercise. 2014;46(5):276-.

27. Menz HB, Dufour AB, Riskowski JL, Hillstrom HJ, Hannan MT. Foot posture, foot function and low back pain: the Framingham Foot Study. Rheumatology. 2013;52(12):2275-82.

28. Ostgaard HC, Andersson GBJ, Karlsson K. Prevalence of Back Pain in Pregnancy. Spine. 1991;16(5):549-52.

29. Ostgaard HC, RoosHansson E, Zetherstrom G. Regression of back and posterior pelvic pain after pregnancy. Spine. 1996;21(23):2777-80.

30. Ponnapula P, Boberg JS. Lower Extremity Changes Experienced During Pregnancy. Journal of Foot \& Ankle Surgery. 2010;49(5):452-8.

31. Schauberger CW, Rooney BL, Goldsmith L, Shenton D, Silva PD, Schaper A. Peripheral joint laxity increases in pregnancy but does not correlate with serum relaxin levels. American Journal of Obstetrics and Gynecology. 1996;174(2):667-71.

32. Segal NA, Boyer ER, Teran-Yengle P, Glass NA, Hillstrom HJ, Yack HJ. Pregnancy Leads to Lasting Changes in Foot Structure. American Journal of Physical Medicine \& Rehabilitation. 2013;92(3):232-40.

33. Stuber J, Zech S, Bay R, Qazzaz A, Richter M. Normative data of the Visual Analogue Scale Foot and Ankle (VAS FA) for pathological conditions. Foot and ankle surgery : official journal of the European Society of Foot and Ankle Surgeons. 2011;17(3):166-72. 
34. Swaddle JP, Witter MS, Cuthill IC. The analysis of fluctuating asymmetry. Animal Behaviour. 1994;48(4):986-9.

35. Vullo V, Richardson J, Hurvitz E. Hip, knee and foot pain during pregnancy and the postpartum period. The Journal of Family Practice. 1996;43(1):63-8.

36. Wearing SC, Hills AP, Byrne NM, Hennig EM, McDonald M. The arch index: A measure of flat or fat feet? Foot \& Ankle International. 2004;25(8):575-81.

37. Wetz H, Hentschel J, Drerup B, Kiesel L, Osada N, Veltmann U. Changes in the Shape and Size of the foot during pregnancy. Orthopade. 2006;11. 


\title{
Appendix A
}

\section{Informed Consent:}

\section{Only Minimal Risk Consent Information and HIPAA Form}

\author{
Principal Investigator \\ McCrory, Jean \\ Department \\ MEDICINE- Exercise Physiology \\ Protocol Number \\ 1509821980 \\ Study Title \\ The Effect of Lower Extremity Asymmetry on Pain During Pregnancy \\ Co-Investigator(s) \\ Casto, Erica; Mancinelli, Corrie
}

\section{Contact Persons}

In the event you experience any side effects or injury related to this research, you should contact Dr. Jean McCrory at (304) 293-0442. (After hours contact: Dr. Jean McCrory at (304) 724-554- 955). If you have any questions, concerns, or complaints about this research, you can contact Dr. Stephen Alway at \{304) 293-0772.

For information regarding your rights as a research subject, to di cuss problems, concerns, or suggestions related to the research, to obtain information or offer input about the research, contact the Office of Research Integrity \& Compliance at (304) 293-7073.

In addition if you would like to discuss problems, concerns, have suggestions related to research, or would like to offer input about the research, contact the Office of Research Integrity and Compliance at 304-293-7073.

\section{Introduction}

You, have been asked to participate in this research study, which has been explained to you by . This study is being conducted by

Dr. Jean McCrory,Phd, Dr. Corrie Mancinelli, PhD, and Erica Casto in the Division of Exercise Physiology at West Virginia University. This study is conducted as part of Erica Casto's thesis requirements for completion of her Bachelor of Science Degree in Exercise Physiology at West Virginia University, under the supervision of Dr. Jean McCrory, PhD.

\section{Purpose(s) of the Study}

The purpose of this study is the learn more about how foot and leg and pelvis alignment differences between the right and left sides are related to pregnancy and how those differences between sides, or asymmetries, are related to pain in the low back and lower extremity during and after pregnancy. WVU expects to enroll approximately 90 subjects ( 30 pregnant pain, 30 pregnant no pain, 30 non-pregnant). 


\section{Description of Procedures}

The study involves us taking measurements on your foot and leg and you answering a series of questionnaires about foot, leg, and low back pain. If you are pregnant, we will ask you to come in to study in the third-trimester as well as post-partum. If you are not pregnant, this will be your only study visit. Each visit will take approximately 45 minutes for you to complete. You will be asked to fill out a questionnaire regarding foot, leg, and low back pain. This will take approximately 10 minutes. You do not have to answer all the questions. You will have the opportunity to see the questionnaire before signing this consent form.

We will ask you to be barefoot so that we can take good measurements of your feet. We will also ask you to wear a close fitting pair of shorts. We will provide you with a pair to wear during testing if you did not bring a pair with you. You can wear any shirt that you like.

Because we would like to study how your foot and leg position may be related to any pain you may feel in your foot, leg, and lower back, we will give you a questionnaire which asks you to rate any discomfort you may be experiencing.

We will measure height and weight using a standard medical scale. We will then use a modified "Beighton's test" to get an overall measure of flexibility, because laxity of the joints is increased during pregnancy. This will include 4 tests of flexibility: hyperextension of knees, hyperextension of elbows, thumb to wrist, and middle finger beyond 90 degrees.

Next, we are going to make a series of measurements about the shape of your foot and the alignments of your ankles and pelvis. We will make measurements of both of your feet. The side we test first (left or right) will be randomly chosen.

Because we think pregnancy may affect length and wide of your foot we will use a caliper, which is similar to a ruler to measure the length and width of your foot.

We also believe that pregnancy may change the shape of the arch of your foot. We will ask you to step on a special inkpad to get a footprint of your foot. This inkpad has a piece of rubber on it that goes between your foot and the ink, so you will not get any ink on your foot. Finally, we are going to measure the height of the arch of your foot using a set of sliding calipers made for this purpose.

Next, we are going to measure the angle that your foot makes with your leg. We will ask you to stand with your feet shoulder width apart, and we will take a picture of the back of your legs.

Then, we will assess foot volume by having you insert your foot into a tank of room temperature water as deep as the middle of you lower leg. We will then refill the tank and repeat with the other foot.

Finally, we will put two reflective stickers, made for use on skin, on front and back of hips. We will take a picture of you standing feet shoulder width apart from the front and back to determine angle that the pelvis makes with the horizontal and assess symmetry between front and back as well as side to side.

In total, 4 pictures will have been taken: two of back of each leg, one of front of pelvis and one of back of pelvis. 
Your name will not appear in the photograph and face will not be included in the pictures. The participation in this study will remain confidential and we will not have any pictures that identify you as a participant in the study.

\section{Discomforts}

There are no known or expected risks from participating in this study, except for the mild frustration associated with answering the questions.

\section{Alternatives}

You do not have to participate in this study.

The only alternative to participating in this study is not participating in this study.

\section{Benefits}

You may not receive any direct benefit from this study. The knowledge gained from this study may eventually benefit others.

\section{Financial Considerations}

There are no special fees for participating in this study. You will be compensated for participation in this study in the form of a gift card.

You will be paid $\$ 20.00$ for each visit, up to a total of $\$ 40.00$. If you withdraw before the end of the study, no additional payments will be made.

\section{Confidentiality}

Any information about you that is obtained as a result of your participation in this research will be kept as confidential as legally possible. Your research records and test results, just like hospital records, may be subpoenaed by court order or may be inspected by the study sponsor or federal regulatory authorities without your additional consent.

In addition, there are certain instances where the researcher is legally required to give information to the appropriate authorities. These would include mandatory reporting of infectious diseases, mandatory reporting of information about behavior that is imminently dangerous to your child or to others, such as suicide, child abuse, etc.

Photographs will be kept locked up and will be destroyed as soon as possible after the research is finished. In any publications that result from this research, neither your name nor any information from which you might be identified will be published without your consent.

\section{HIPAA}

We know that information about you and your health is private. We are dedicated to protecting the privacy of that information. Because of this promise, we must get your written authorization (permission) before we may use or disclose your protected health information or share it with others for research purposes. 
You can decide to sign or not to sign this authorization section. However, if you choose not to sign this authorization, you will not be able to take part in the research study. Whatever choice you make about this research study will not have an effect on your access to medical care.

\section{Persons/Organizations Providing the Information}

Patient/West Virginia University Hospitals

\section{Persons/Organizations Receiving the Information}

- $\quad$ The research site(s) carrying out this study. This includes UHA or UHA Affiliated, WVU, WVU Hospitals. It also includes each site's research staff and medical staff

- $\quad$ Health care providers who provide services to you as part of this research study.

- Laboratories and other people and groups that look into your health information as part of this study in agreement with the study protocol.

- $\quad$ The United State Department of Health and Human Services (which includes the National Institutes of Health (NIH), Food and Drug Administration (FDA)) and other groups that have the right to use the information as required by law.

- $\quad$ The members and staff of any Institutional Review Board (IRB) that oversees this research study.

- $\quad$ West Virginia University Office of Research Compliance and Office of Sponsored Programs.

- West Virginia University Clinical Trials Research Unit.

\section{The Following Information Will Be Used}

Information from your existing medical records and new information about you that is created or collected during the study such as: history and physicals, clinic visit notes, nursing and staff notes, laboratory results, $x$ rays, EKG results, demographic data, pulmonary tests, imaging scans and study forms.

\section{The Information is Being Disclosed for the Following Reasons}

- $\quad$ Review of your data for quality assurance purposes

- $\quad$ Publication of study results (without identifying you)

- $\quad$ Other research purposes such as reviewing the safety or effectiveness of the study drug and other products or therapies; conducting performance reviews of the study drug; evaluating other products or therapies for patients; developing a better understanding of disease; improving the design of future clinical trials

\section{You May Cancel this Authorization at Any Time by Writing to the Principal Investigator}

Jean L. McCrory, PhD, 8315 HSC South,

PO Box 9227,

Morgantown, WV 26506-9227;

email: jlmccrory@hsc.wvu.edu 
If you cancel this authorization, any information that was collected already for this study cannot be withdrawn. Once information is disclosed, according to this authorization, the recipient may redisclose it and then the information may no longer be protected by federal regulations.

You have a right to see and make copies of your medical records. You will not be able to see or copy your records related to the study until the sponsor has completed all work related to the study. At that time you may ask to see the study doctor's files related to your participation in the study and have the study doctor correct any information about you that is wrong.

This authorization will expire at the end of the study unless you cancel it before that time (or has a specific expiration date).

\section{Voluntary Participation}

Participation in this study is voluntary. You are free to withdraw your consent to participate in this study at any time.

Refusal to participate or withdrawal will not affect [your class standing or grades, as appropriate] and will involve no penalty to you. Refusal to participate or withdrawal will not affect your future care, or your employee status at West Virginia University.

In the event new information becomes available that may affect your willingness to participate in this study, this information will be given to you so that you can make an informed decision about whether or not to continue your participation.

You have been given the opportunity to ask questions about the research, and you have received answers concerning areas you did not understand.

Upon signing this form, you will receive a copy.

I willingly consent to participate in this research.

\section{Signatures}

Signature of Subject

Printed Name

Date

Time

The participant has had the opportunity to have questions addressed. The participant willingly agrees to be in the study. 
Signature of Investigator or Co-Investigator

Printed Name

Date

Time 


\title{
Appendix B
}

\section{Subject Questionnaire}

\author{
Subject \#: \\ Visit \#: \\ Date:
}

\section{Subject Questionnaire}

Foot Alignment in Pregnancy

\section{General:}

1. Age:

2. Medications:

3. Does someone help you with tasks around the house?

Yes

No

4. Are there stairs in your home?

Yes

No

a. If yes, how often do you climb them?

times/day

5. What type of shoes do you normally wear? (i.e. sneakers, heels, boots)

6. Do you have a known leg length discrepancy? Yes No

7. Are you pregnant with more than one baby? Yes No 


\section{Exercise Habits:}

8. Do you currently exercise? Yes No If yes:

a. How often? times/week

b. Duration of exercise session:

c. What type of exercise?

\begin{tabular}{|l|l|l|}
\hline Activity & Frequency (days/week) & Duration \\
\hline $\begin{array}{l}\text { Team sport (please } \\
\text { specify): }\end{array}$ & & \\
\hline Walking & & \\
\hline Jogging/running & & \\
\hline Swimming & & \\
\hline Bicycling & & \\
\hline Skating & & \\
\hline Martial arts & & \\
\hline Tai chi & & \\
\hline Aerobics & & \\
\hline Dancing & & \\
\hline Strength/ weight training & & \\
\hline Yoga & & \\
\hline Other (please describe): & & \\
& & \\
\hline
\end{tabular}

9. If pregnant: Was this exercise regimen started before pregnancy? Yes No

Page 2 of 3 


\section{Daily tasks:}

10. How many hours per day do you spend standing?

11. How many hours per day do you spend sitting?

12. Do daily tasks often require lifting objects?

a. If yes, how much weight is lifted?

b. How often? (times/day)

13. If pregnant: Have your work/home responsibilities changed since becoming pregnant? Yes No

a. If yes, what accommodations have been made?

\section{Pain History:}

For pregnant women:

14. Did you experience foot pain prior to pregnancy? Yes No

15. Did you experience low back pain prior to pregnancy? Yes No

16. Did you experience pain in the hip/buttocks area prior to pregnancy? Yes No If the answer to 14,15 or 16 is yes,

17. Has this pain changed since pregnancy?

\section{Decreased Stayed the same Increased}

\section{Falls:}

14. Did you experience any loss of balance resulting in a fall where some part of your body - other than your feet - touched the ground?

If pregnant: During pregnancy: Yes No
Within the year prior to pregnancy: Yes No
If not pregnant: Within the past year: Yes No

If any of the answers on Question 14 are yes, please fill out Fall Incident

\section{Information Form}

Page 3 of 3 


\section{Visual Analogue Scale}

\section{Pregnancy and Foot Study: Lower Extremity Pain Visual Analogue Scale}

On the following pages is a questionnaire with questions related to lower body problems (eg. Pain). These include the lower back, hip/buttocks, upper leg, knee, lower leg and foot/ankle. To answer the questions a scale is available in the form of a line. Please mark the appropriate point on the line with a cross, which describes best your personal situation. At the very left side of the line is the most negative value, at the very right the most positive. Please use only marks, do not write text.

This is an example for an answer of the question "How are you today?" as shown:

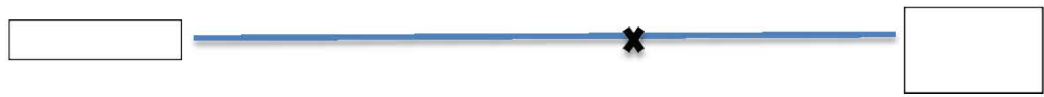

The answer at the cross on the line means in this example that you feel "well", however not "verywell".

For any area of pain that applies to you, please indicate with a dot or dots in the red highlighted area of the diagram where exactly you experience pain.

You do not have to answer each question! Answer only the questions which you would like and which you have understood! Please use the field "additions/characeristics/remarks" for suggestions for improvement and/or criticism.

Explanation of someterms:

Physical rest: This means that you do not do arduous things, i.e. you are reading a paper, lying on the sofa or in a bed, watching television etc.

Physical activity: This means that you are performing physically demanding activities, i.e. arduous garden work, occupational work, sport etc.

Activities of daily life: Personal activities such as getting out of bed, eating, washing yourself, getting dressed, tying your shoes etc.

\section{Additions / remarks}




\section{LOWERBACK}

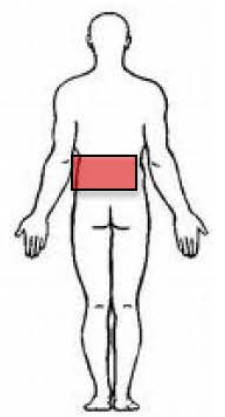

How much does lower back pain affect your gait?

Stronglimping
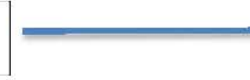

Never, very

How often do you have lower back pain in physical rest?

\begin{tabular}{l|}
\hline $\begin{array}{l}\text { Constantly, } \\
\text { always }\end{array}$ \\
\hline
\end{tabular}

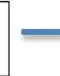

How intense is this back pain in physical rest?

Extreme pain

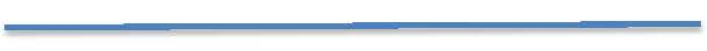

No pain

How often do you have lower back pain during physical activity?

\section{Constantly,}

always

Never, very

rarely

How strong is this lower back pain during physical activity?

Stronglimping
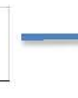

How much do lower back problems affect your daily activities (eg. getting dressed, eating, washing, etc.)?

\begin{tabular}{|l|l|l|}
$\begin{array}{l}\text { Impossible on } \\
\text { my own, need } \\
\text { constanthelp }\end{array}$ & $=\begin{array}{l}\text { No limitation } \\
\text { dimbing } \\
\text { stairs }\end{array}$ \\
\hline
\end{tabular}


HIP/BUTTOCKS

LEFT

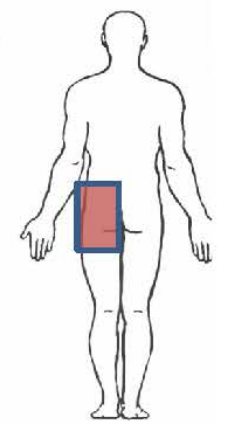

How much does hip/buttocks pain affect your gait?

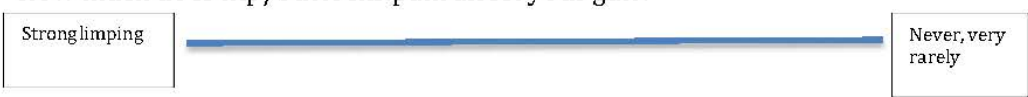

How often do you have hip/buttocks pain in physical rest?

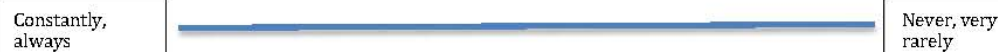

\begin{tabular}{lll} 
always & rarely \\
\hline
\end{tabular}

How intense is this hip/buttocks in physical rest?

Extreme pain

No pain

How often do you have hip/buttocks pain during physical activity?

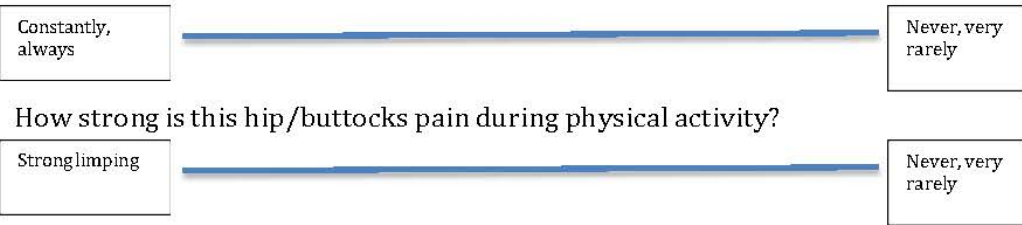

How much do hip/buttocks p roblems affect your daily activities (eg. getting dressed, eating, washing, etc.)?

Impossible on

my own, need

constanthelp

No limitation

dimbling

stairs 


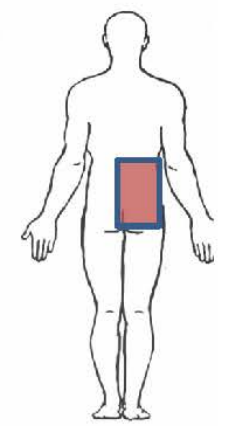

How much does hip/buttocks pain affect your gait?

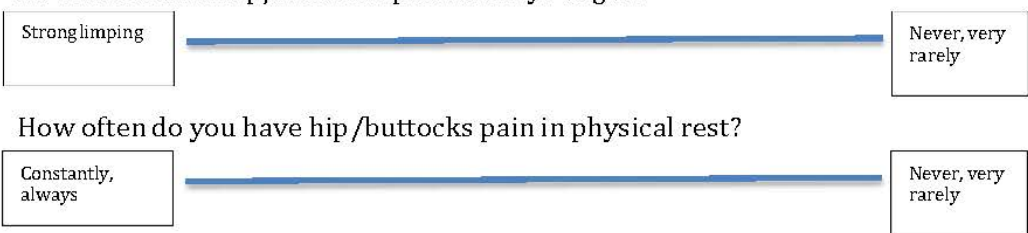

How intense is this hip/buttocks in physical rest?

Extreme pain

No pain

How often do you have hip/buttocks pain during physical activity?

Constantly,

always

Never, very

How strong is this hip/buttocks pain during physical activity?

Stronglimping

Never, very

rarely

How much do hip/buttocks problems affect your daily activities (eg. getting dressed, eating, washing, etc.)?

Impossible on

my own, need

No limitation

climbling

constanthelp

stairs 
IJPPFR I.F.C

LEFT

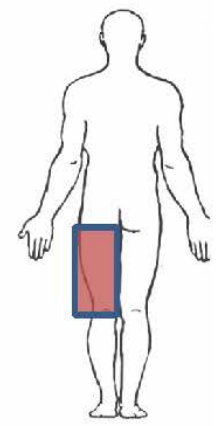

How much does upper leg pain affect your gait?

Stronglimping

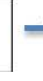

Never, very

rarely

How often do you have upper leg pain in physical rest?

Constantly,
always

Never, very

rarely

How intense is this upper leg in physical rest?

Extreme pain

No pain

How often do you have upper leg pain during physical activity?

Constantly,

always

Never, very

rarely

How strong is this upper leg pain during physical activity?

Strong limping

Never, very

How much do upper leg problems affect your daily activities (eg. getting dressed, eating, washing, etc.)?

Impossible on

my own, need

constanthelp

No limitation

climbing

stairs 
IJPPE.R I.F.C

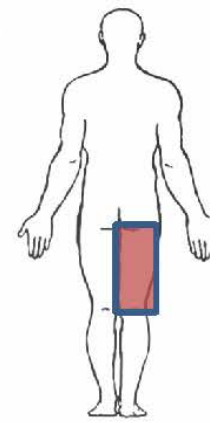

RIGHT

How much does upper leg pain affect your gait?

Strong limping

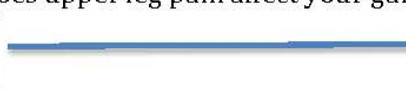

Never, very

rarely

How often do you have upper leg pain in physical rest?

Constantly,
always

Never, very rarely

How intense is this upper leg in physical rest?

Extreme pain No pain

How often do you have upper leg pain during physical activity?

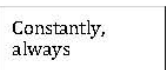

Never, ver rarely

How strong is this upper leg pain during physical activity?

Stronglimping

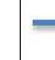

How much do upper leg problems affect your daily activities (eg. getting dressed, eating, washing, etc.)?

Impossible on

my own, need

Nolimitation

constanthelp

climbing
stairs 


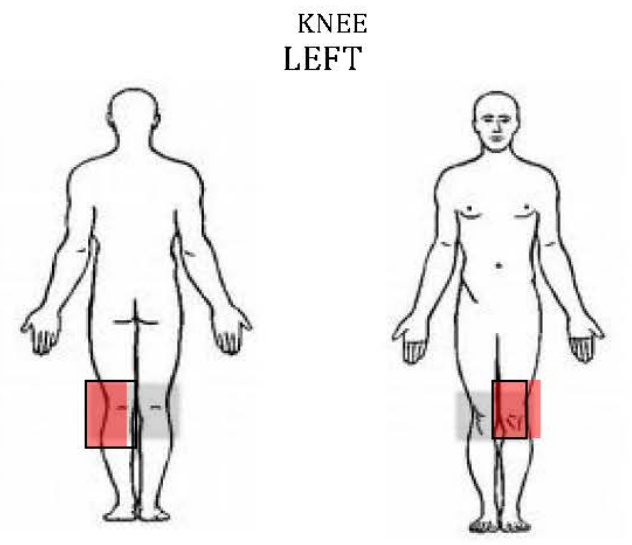

How much does knee pain affect your gait?

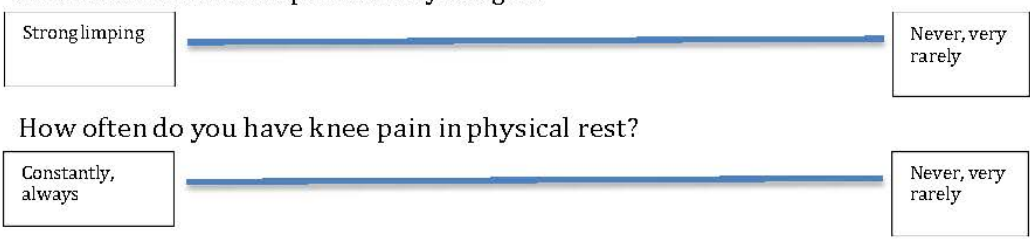

How intense is this knee in physical rest?

Extreme pain No pain

How often do you have knee pain du ring physical activity?

Constantly,

always

Never, very

rarely

How strong is this knee pain during physical activity?

Stronglimping

Never, very

rarely

How much do knee problems affect your daily activities (eg. getting dressed, eating, washing, etc. )?

Impossible on

my own, need

constanthelp

No limitation

climbing

stairs 


\section{KNEE}

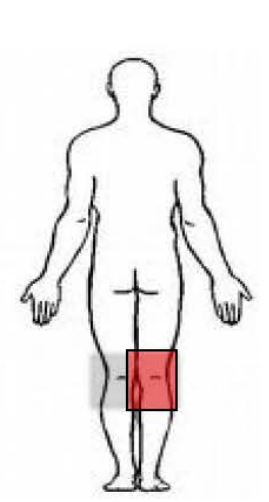

\section{RIGHT}

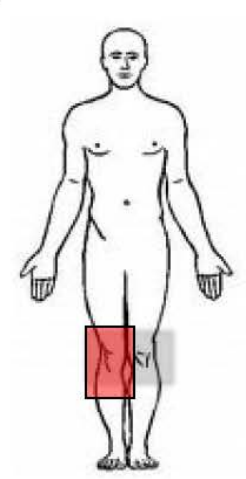

How much does knee pain affect your gait?

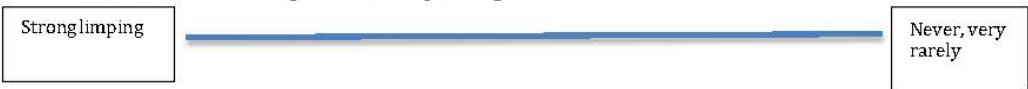

How often do you have knee pain in physical rest?

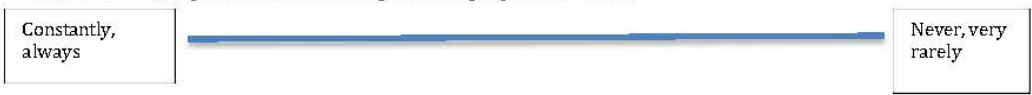

How intense is this knee in physical rest?

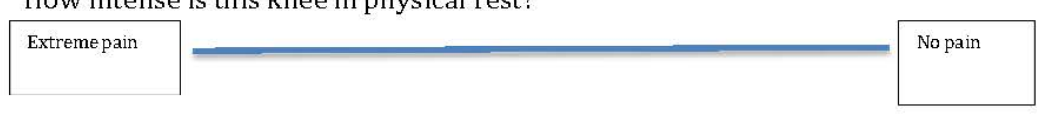

How often do you have knee pain during physical activity?

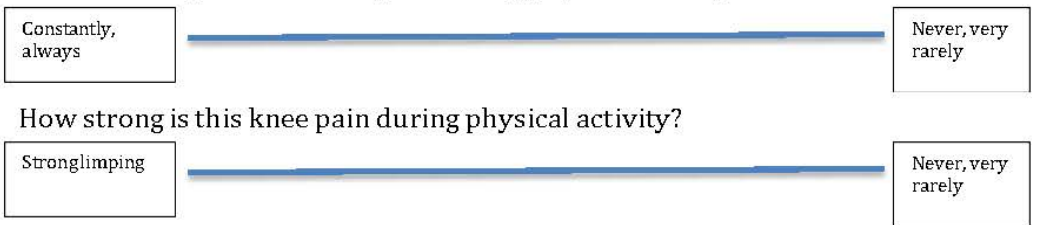

How much do knee problems affect your daily activities (eg. getting dressed, eating, washing, etc. )?

Impossible on

my own, need

constanthelp

Nolimitation

climbing

stairs 


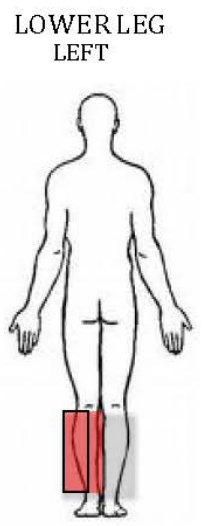

How much does lower leg pain affect your gait?

Stronglimping

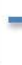

Never, very

rarely

How often do you have lower leg pain in physical rest?

Constantly,
always

Never, very

rarely

How intense is this lower leg in physical rest?

Extreme pain

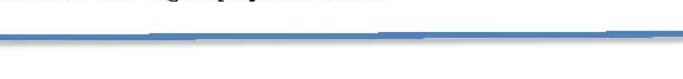

No pain

How often do you have lower leg pain during physical activity?

Constantly,

always

Never, very

rarely

How strong is this lower leg pain during physical activity?

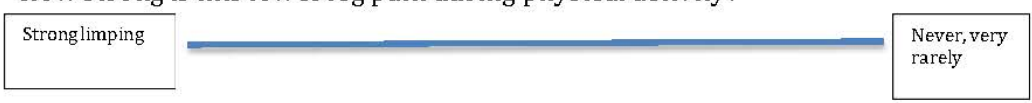

How much do lower leg problems affect your daily activities (eg. getting dressed, eating, washing, etc.)?

Impossible on

my own need

constanthelp

No limitation

climbing

stairs 


\section{LOWER LEG \\ RIGHT}

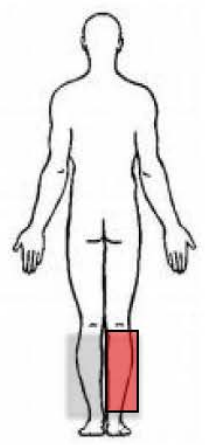

How much does lower leg pain affect your gait?

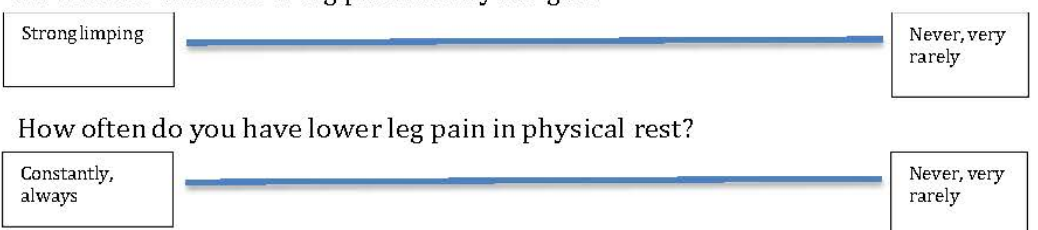

How intense is this lower leg in physical rest?

Extreme pain

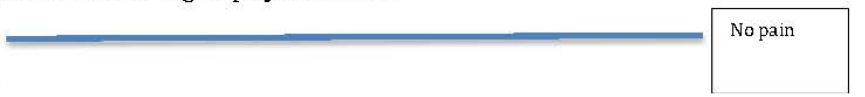

How often do you have lower leg pain during physical activity?

Constantly,

Constan

Never, very

rarely

How strong is this lower leg pain during physical activity?

Stronglimping

Never, very

rarely

How much do lower leg problems affect your daily activities (eg. getting dressed, eating, washing, etc.)?

Impossible on

my own, need

No limitation

dimbing

constanthelp

stairs 


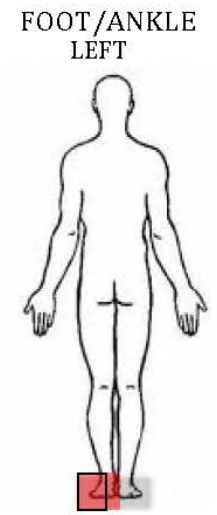

How much does foot/ankle pain affect your gait?

Stronglimping

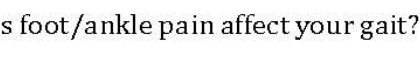

How often do you have foot/ankle pain in physical rest?

\begin{tabular}{|l|}
\hline $\begin{array}{l}\text { Constantly, } \\
\text { always }\end{array}$ \\
\hline
\end{tabular}

-
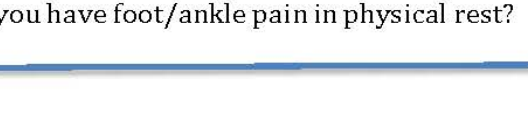

How intense is this foot/ankle in physical rest?

Extreme pain
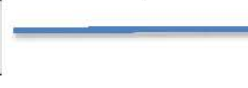

How often do you have foot/ankle pain during physical activity?

Constantly,

always

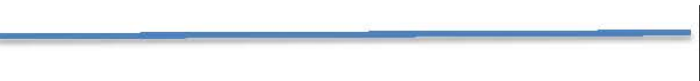

Never, very

rarely

How strong is this foot/ankle pain during physical activity?

Stronglimping

Never, very

rarely

How much do foot/ankle problems affect your daily activities (eg. getting dressed, eating, washing, etc.)?

\begin{tabular}{|l|l|l|}
\hline $\begin{array}{l}\text { Impossible on } \\
\text { my own, need } \\
\text { constant help }\end{array}$ & $\begin{array}{l}\text { No limitation } \\
\text { dimbing } \\
\text { stairs }\end{array}$ \\
\hline
\end{tabular}


FOOT/ANKLE

RIGHT

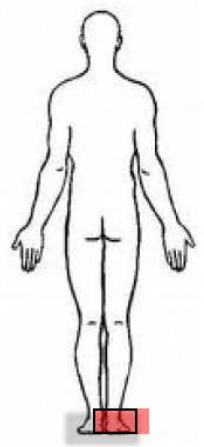

How much does foot/ankle pain affect your gait?

Stronglimping

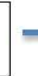

rarely

How often do you have foot/ankle pain in physical rest?

Constantly,
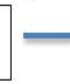

Never, very

always

How intense is this foot/ankle in physical rest?

Extreme pain No pain

How often do you have foot/ankle pain during physical activity?

\section{Constantly,}

always

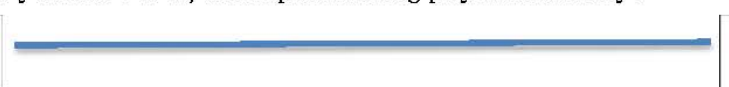

Never, very rarely

How strong is this foot/ankle pain during physical activity?

$$
\text { Stronglimping }
$$

How much do foot/ankle problems affect your daily activities (eg. getting dressed, eating, washing, etc.)?

\begin{tabular}{|l|l|l|}
$\begin{array}{l}\text { Impossible on } \\
\text { my own, need } \\
\text { constanthelp }\end{array}$ & $\begin{array}{l}\text { No limitation } \\
\text { dimbing } \\
\text { stairs }\end{array}$ \\
\hline
\end{tabular}




\section{Appendix C}

\section{Data Collection Sheet: Measurement Chart}

\section{FOOT ALIGNMENT IN PREGNANCY: MEASUREMENT CHART}

Subject:

Date:

Visit:

Weeks pregnant:

Age:

Height:

Weight:

BMI:

Pre-pregnancy weight: Weight gained:

\section{BEIGHTONS TEST:}

Knees bent backward:

Elbows bent backwards:

Thumbs to forearm:

Little fingers beyond $90^{\circ}$

TOTAL:

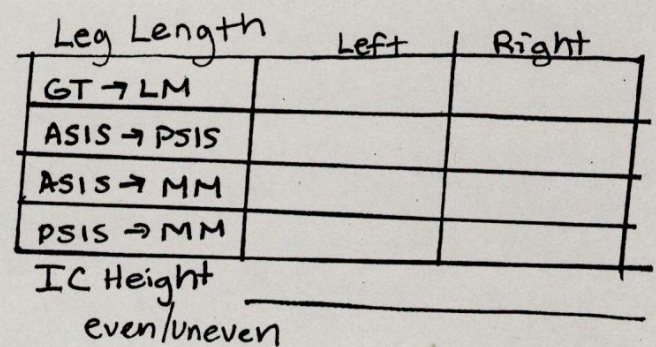

BIOMECHANICAL MEASUREMENTS

\begin{tabular}{|c|c|c|c|c|}
\hline & \multicolumn{4}{|c|}{ Unweighted } \\
\hline & \multicolumn{2}{|l|}{ Left } & \multicolumn{2}{|c|}{ Right } \\
\hline \multicolumn{5}{|l|}{ Leg length $(\mathrm{cm})$} \\
\hline \multicolumn{5}{|l|}{ Foot length (cm) } \\
\hline \multicolumn{5}{|l|}{ Foot width $(\mathrm{cm})$} \\
\hline \multirow{2}{*}{\multicolumn{5}{|c|}{$\begin{array}{l}\text { Total area (pixels?) } \\
\text { Midfoot area (pixels) }\end{array}$}} \\
\hline & & & & \\
\hline \multirow{3}{*}{$\begin{array}{l}\text { FL } \\
\text { TFL } \\
\text { FH }\end{array}$} & Sit & Stand & Sit & Stand \\
\hline & & & & \\
\hline & & & & \\
\hline
\end{tabular}

\begin{tabular}{|l|l|l|l|l|}
\hline Calf angle $\left({ }^{\circ}\right)$ & & & & \\
\hline Heel angle $\left(^{\circ}\right)$ & & & & \\
\hline Pelvic Obliquity $\left(^{\circ}\right)$ & & & & \\
\hline
\end{tabular}




\section{Appendix D}

\section{Normality Tests: Shapiro-Wilk}

Tests of Normality

\begin{tabular}{|l|r|r|r|r|r|r|}
\hline & \multicolumn{3}{|c|}{ Kolmogorov-Smirnov $^{\mathrm{a}}$} & \multicolumn{3}{|c|}{ Shapiro-Wilk } \\
\cline { 2 - 7 } & Statistic & $\mathrm{df}$ & \multicolumn{1}{c|}{ Sig. } & Statistic & $\mathrm{df}$ & \multicolumn{1}{c|}{ Sig. } \\
\hline FL_Diff & .230 & 18 & .013 & .911 & 18 & .090 \\
FW_Diff & .176 & 18 & .145 & .960 & 18 & .606 \\
Al_Diff & .099 & 18 & $.200^{*}$ & .961 & 18 & .612 \\
AHI_Stand_Diff & .133 & 18 & $.200^{*}$ & .952 & 18 & .454 \\
ARIDiff & .098 & 18 & $.200^{*}$ & .960 & 18 & .594 \\
ADDiff & .144 & 18 & $.200^{*}$ & .967 & 18 & .730 \\
RFA_Diff & .129 & 18 & $.200^{*}$ & .959 & 18 & .591 \\
\hline
\end{tabular}

*. This is a lower bound of the true significance.

a. Lilliefors Significance Correction 